\title{
A FREE BOUNDARY PROBLEM DESCRIBING THE SATURATED-UNSATURATED FLOW IN A POROUS MEDIUM
}

\author{
GABRIELA MARINOSCHI
}

Received 15 September 2003

This paper presents a functional approach to a nonlinear model describing the complete physical process of water infiltration into an unsaturated soil, including the saturation occurrence and the advance of the wetting front. The model introduced in this paper involves a multivalued operator covering the simultaneous saturated and unsaturated flow behaviors and enhances the study of the displacement of the free boundary between these two flow regimes. The model resides in Richards' equation written in pressure form with an initial condition and boundary conditions which in this work express the inflow due to the rain on the soil surface on the one hand, and characterize a certain permeability corresponding to the underground boundary, on the other hand. Existence, uniqueness, and regularity results for the transformed model in diffusive form, that is, for the moisture of the soil, and the existence of the weak solution for the pressure form are proved in the $3 \mathrm{D}$ case. The main part of the paper focuses on the existence of the free boundary between the saturated and unsaturated parts of the soil, and this is proved, in the 1D case, for certain stronger assumptions on the initial data and boundary conditions.

\section{Introduction}

The paper has the purpose of introducing a mathematical model able to describe the complete phenomenon of water infiltration into an unsaturated soil, the evolution of soil moisture up to saturation, and the advance of the interface between the saturated and unsaturated regions. From the hydraulic point of view the problem relies on the Darcian flow of an incompressible fluid in an isotropic, homogeneous porous medium with a constant porosity in the absence of evaporation. Under certain conditions depending on the rate at which rain water is supplied, the initial moisture distribution in the soil, the presence of underground sources, and the boundary permeability, the saturation of the ground surface could occur at the so-called saturation time. Consequently a waterfront starts to move downwards and this represents the unknown interface between the saturated and unsaturated flow regimes. In these problems the hydraulic functions empirically introduced by soil scientists, but characterizing with good results the hydraulics 
properties of various soils, raise a difficult mathematical problem: when the moisture of the soil comes close to the saturation value, the diffusivity expressed as a function of moisture blows up, and so saturation could not be mathematically described, see, for example, [14]. Correspondingly, another hydraulic function standing for a coefficient of the equation written in pressure form vanishes when pressure tends to zero and forces this equation to degenerate. In the mathematical literature devoted to this subject, this particularity was avoided by considering a finite valued diffusivity, see $[1,2,9,12,13]$. In [1], for instance, a time-dependent saturated-unsaturated flow was treated in the case of time-dependent water levels and an existence result for the corresponding weak formulation was proved. More recently, in [8], a model of the saturated-unsaturated flow lying on a special definition of the boundary conditions that changes during the phenomenon evolution, has been developed also for a finite value of the diffusivity at saturation.

In $[5,6,11]$ the model of unsaturated infiltration with a blowing up diffusivity was treated in the framework of the semigroup theory.

By the model introduced in this paper the difficulty arisen at the interface between the saturated and unsaturated regimes is surpassed, and so the complete mathematical description of the saturated-unsaturated flow is enabled. Starting from the classical model using Richards' equation, the definition of the weak solution in pressure form is given and the connection with the saturated-unsaturated model is made. Then, passing from pressure to moisture by the aid of the hydraulic functions, a specific model is stated by introducing a multivalued operator that characterizes the behaviors at the saturatedunsaturated interface. Using an approximating model, that provides necessary results, the existence and uniqueness of the solution to this specific model and the existence of the weak solution are proved in the 3D case. However, the uniqueness of the weak solution (in pressure form) does not follow under the hypotheses used up to now, and this requires first the assurance of the free boundary existence. Imposing stronger assumptions, some supplementary regularity properties of the solution to the approximating problem are found and these enable the proof of the existence of the free boundary in the 1D case. Finally, the uniqueness of the weak and smooth solution is proved.

\section{The mathematical model}

The behaviors of an unsaturated porous soil, so partially filled with water, is completely known from the hydraulic point of view if two functions are given: one is the hydraulic conductivity $k$ and the other is the constitutive relationship linking the volumetric water content, or moisture of the soil $\theta$, with the pressure head $h$. They depend nonlinearly on $h$.

Since an unsaturated soil is characterized by convenience by negative pressure $(h<0)$ (see [7]), these functions are defined as follows.

$\left(\mathrm{a}_{1}\right) k:\left[h_{r}, 0\right) \rightarrow\left[K_{r}, K_{s}\right) ; \theta:\left[h_{r}, 0\right) \rightarrow\left[\theta_{r}, \theta_{s}\right)$, and they are positive and differentiable, with $h_{r}<0$.

The value $\theta_{s}$ is the value of moisture at saturation and $\theta_{r}=\theta\left(h_{r}\right)$ is the residual value of moisture, meaning the moisture resident in a dried soil. From practice it is known that after all drainage forces have ceased, a soil retains a small amount of water which does no longer enter in the water circulation, so it follows that $\theta_{r}>0$. The values $K_{s}$ and $K_{r}$ are 
the values of conductivity at saturation and for a dried soil, respectively. The positive values $\theta_{r}, \theta_{s}, K_{r}$, and $K_{s}$ are soil characteristics and they are known.

Generally, this type of processes displays a hysteretic behavior, especially when a cycle of wetting and drying processes happens. But if we assume that only one process takes place, for example, infiltration, then we can disregard the hysteretic aspect (see [7]).

So we may consider that

$\left(\mathrm{a}_{2}\right) h \rightarrow \theta(h)$ and $h \rightarrow k(h)$ are single-valued and monotonically increasing functions on $\left[h_{r}, 0\right)$.

The derivative of $\theta$ with respect to $h$ is called water capacity $C$ and

$\left(\mathrm{a}_{3}\right) h \rightarrow C(h)$ is a positive, bounded, continuous, and monotonically decreasing function on $\left[h_{r}, 0\right)$.

We also assume the following property:

$\left(\mathrm{i}_{K}\right)$ there exists $M>0$ such that $k^{\prime}(h) \leq M C(h)$.

When a part of the soil begins to saturate itself, the pressure within it becomes positive, $h \geq 0$, and all these functions take constant values, namely,

$\left(\mathrm{a}_{4}\right) \theta(h)=\theta_{s}, k(h)=K_{s}$, and $C(h)=0$, for $h \geq 0$.

We are now ready to formulate the mathematical model of infiltration of an incompressible fluid (water) into an isotropic and homogeneous porous medium with a constant porosity.

Let $\Omega$ be an open bounded subset of $\mathbb{R}^{N}(N=1,2,3)$ with the boundary $\partial \Omega \stackrel{\text { notation }}{=} \Gamma$ sufficiently smooth (e.g., a piecewise $(N-1)$-dimensional manifold of class $\left.C^{2}\right)$, let $(0, T)$ be a finite time interval, and let $x \in \Omega$ represent the vector $x=\left(x_{1}, x_{2}, x_{3}\right)$. To be more specific, we will consider $\Omega$ to be the cylinder $\Omega=\left\{x ;\left(x_{1}, x_{2}\right) \in D, 0<x_{3}<L\right\}$, where $D$ is an open bounded subset of $\mathbb{R}^{N-1}$ with smooth boundary. We consider that $\Gamma$ is composed of the disjoint boundaries $\Gamma_{u}, \Gamma_{\text {lat }}$, and $\Gamma_{b}$, all sufficiently smooth, and define $\Gamma_{u}=\{x \in$ $\left.\Gamma ; x_{3}=0\right\}, \Gamma_{b}=\left\{x \in \Gamma ; x_{3}=L\right\}$, and $\Gamma=\Gamma_{u} \cup \Gamma_{\text {lat }} \cup \overline{\Gamma_{b}}$. We also denote $\Gamma_{\alpha}=\Gamma_{\text {lat }} \cup \overline{\Gamma_{b}}$, where $\Gamma_{u} \cap \Gamma_{\alpha}=\varnothing$. Correspondingly, we define $\Sigma_{\alpha}=\Gamma_{\alpha} \times(0, T), \Sigma_{u}=\Gamma_{u} \times(0, T), \Sigma_{b}=$ $\Gamma_{b} \times(0, T)$, and $\Sigma_{\text {lat }}=\Gamma_{\text {lat }} \times(0, T)$.

The mathematical model describing the water infiltration into a soil with the properties specified above consists of Richards' equation written for the pressure head $h(x, t)$ (see [7]), with an initial condition and various boundary conditions. In this study we will take into account a more realistic situation, in which infiltration is produced by a rainfall (or irrigation) on the surface of the soil $\left(\Gamma_{u}\right)$ and the domain considered has a kind of semipermeable boundary $\left(\Gamma_{\alpha}\right)$. The model reads

$$
\begin{gathered}
C(h) \frac{\partial h}{\partial t}-\nabla \cdot(k(h) \nabla h)+\frac{\partial k(h)}{\partial x_{3}}=f \quad \text { in } Q=\Omega \times(0, T), \\
h(x, 0)=h_{0}(x) \quad \text { in } \Omega, \\
q \cdot v=u(x, t) \quad \text { on } \Sigma_{u}, \\
q \cdot v=\alpha(x) K^{*}(h)+f_{0}(x, t) \quad \text { on } \Sigma_{\alpha} .
\end{gathered}
$$


By $q$ we denoted the flux defined by $q(x, t)=k(h) i_{3}-k(h) \nabla h$, where $v$ is the outward normal to $\Gamma$ and $i_{3}$ is the unit vector along $O x_{3}$, directed downwards, and $\alpha$ is a bounded, continuous, and positive function on $\Gamma_{\alpha}$. The function $K^{*}$ is a primitive of $k$ that will be specified later.

For our mathematical purposes, we extend the functions $C$ and $k$ by continuity to the left of $h_{r}$ such that $0<C(h) \leq C_{r}, 0<k(h) \leq K_{r}$, and

$$
\lim _{h \rightarrow-\infty} \frac{k(h)}{C(h)}=\frac{K_{r}}{C_{r}}=\rho>0, \quad \lim _{h \rightarrow-\infty} k(h)=\lim _{h \rightarrow-\infty} C(h)=0 .
$$

To accomplish this we may define $k$ and $C$ by

$$
k(h)=K_{r} \exp \left(h-h_{r}\right), \quad C(h)=C_{r} \exp \left(h-h_{r}\right) \quad \text { for } h<h_{r}, K_{r} \leq M C_{r} .
$$

Hence we consider the functions $C$ and $k$ defined on $\mathbb{R}$ with properties $\left(a_{1}\right),\left(a_{2}\right),\left(a_{3}\right)$, and $\left(\mathrm{a}_{4}\right)$ and (2.2).

We define the primitives of $C$ and $K$ by

$$
\begin{aligned}
C^{*}(h) & = \begin{cases}\theta_{r}+\int_{h_{r}}^{h} C(\zeta) d \zeta, & h<0, \\
\theta_{s}, & h \geq 0,\end{cases} \\
K^{*}(h) & = \begin{cases}\int_{-\infty}^{h} k(\zeta) d \zeta, & h<0, \\
K_{s}^{*}+K_{s} h, & h \geq 0, K_{s}^{*}=K^{*}(0),\end{cases}
\end{aligned}
$$

and denote $\theta=C^{*}(h)$. We notice that

$$
C^{*}:(-\infty, \infty) \longrightarrow\left(\theta_{\min }, \theta_{s}\right], \quad \text { where } \theta_{\min }=\lim _{h \rightarrow-\infty} C^{*}(h)=\theta_{r}-C_{r}
$$

Both functions are continuous and monotonically increasing $\left(C^{*}\right.$ on $\left.h<0\right)$ and with these notations the model becomes

$$
\begin{gathered}
\frac{\partial C^{*}(h)}{\partial t}-\Delta K^{*}(h)+\frac{\partial k(h)}{\partial x_{3}}=f \quad \text { in } Q, \\
h(x, 0)=h_{0}(x) \quad \text { in } \Omega, \\
q \cdot v=u(x, t) \quad \text { on } \Sigma_{u}, \\
q \cdot v=\alpha(x) K^{*}(h)+f_{0}(x, t) \quad \text { on } \Sigma_{\alpha} .
\end{gathered}
$$




\section{Weak solution}

Let $V$ be the space $H^{1}(\Omega)$ endowed with the usual Hilbertian norm.

Definition 3.1. The function $h \in L^{2}\left(0, T ; L^{2}(\Omega)\right)$ is said to be a weak solution to problem (2.7) if $K^{*}(h) \in L^{2}(0, T ; V)$ and

$$
\begin{aligned}
\int_{Q}(- & \left.C^{*}(h) \phi_{t}(x, t)+\nabla K^{*}(h) \cdot \nabla \phi(x, t)-k(h) \frac{\partial \phi}{\partial x_{3}}(x, t)\right) d x d t \\
= & \int_{\Omega} \phi(x, 0) C^{*}\left(h_{0}(x)\right) d x-\int_{\Sigma_{\alpha}}\left(\alpha(x) K^{*}(h)+f_{0}(x, t)\right) \phi(x, t) d \sigma d t \\
& -\int_{\Sigma_{u}} u(x, t) \phi(x, t) d \sigma d t+\int_{Q} f(x, t) \phi(x, t) d x d t
\end{aligned}
$$

for all $\phi \in L^{2}(0, T ; V)$ with $\phi_{t} \in L^{2}\left(0, T ; L^{2}(\Omega)\right)$ and $\phi(x, T) \equiv 0$.

In (3.1) $d x$ is the Lebesgue measure and $d \sigma$ is the surface measure. Further, if any confusion is avoided, we will no longer indicate in the integrands those function arguments which represent the integration variables.

Also we will denote by $(\cdot, \cdot)$ and $\|\cdot\|$ the scalar product and the norm in $L^{2}(\Omega)$, respectively.

Suppose that the domain $Q$ is divided into two very well delimited domains, corresponding to the saturated and unsaturated parts of the soil and the saturated part is above. Denote by $Q_{-}=\{(x, t) ; h(x, t)<0\}$ the unsaturated part, $Q_{+}=\{(x, t) ; h(x, t)>0\}$ the saturated region, and $Q_{0}=\{(x, t) ; h(x, t)=0\}$ the free surface (boundary) separating the saturated part $Q_{+}$from the unsaturated one, $Q_{-}$. We specify that $\nu$ signifies the normal to a boundary, no matter which boundary is in discussion, but we should keep in mind that the respective normal is always directed to the exterior of the domain delimited by that boundary. However, in order to avoid any confusion, we will mark by superscripts the normals to the interface, that is, by $\nu^{+}$we mean the normal to $Q_{0}$ directed towards $Q_{-}$, and by $v^{-}$the normal to $Q_{0}$ directed to $Q_{+}$, and we notice that $\nu^{+}=-v^{-}$. Moreover, we denote

$$
h^{-}\left(x_{0}, t_{0}\right)=\lim _{\substack{(x, t) \rightarrow\left(x_{0}, t_{0}\right) \\(x, t) \in Q_{-}}} h(x, t), \quad q^{-}\left(x_{0}, t_{0}\right)=-\lim _{\substack{(x, t) \rightarrow\left(x_{0}, t_{0}\right) \\(x, t) \in Q_{-}}}\left(q \cdot v^{-}\right)(x, t)
$$

Similarly, by

$$
h^{+}\left(x_{0}, t_{0}\right)=\lim _{\substack{(x, t) \rightarrow\left(x_{0}, t_{0}\right) \\(x, t) \in Q_{+}}} h(x, t), \quad q^{+}\left(x_{0}, t_{0}\right)=\lim _{\substack{(x, t) \rightarrow\left(x_{0}, t_{0}\right) \\(x, t) \in Q_{+}}}\left(q \cdot v^{+}\right)(x, t),
$$

we denote the corresponding right-hand side limits. We recall that $O x_{3}$ is directed downwards. 
Proposition 3.2. If $h$ is a weak, smooth solution to (2.7), then $h$ is a solution to the following model describing the water infiltration into an unsaturated-saturated soil:

$$
\begin{gathered}
C(h) \frac{\partial h}{\partial t}-\Delta K^{*}(h)+\frac{\partial k(h)}{\partial x_{3}}=f \quad \text { in } Q_{-}, \\
-K_{s} \Delta h=f \quad \text { in } Q_{+}, \\
h(x, 0)=h_{0}(x) \quad \text { in } \Omega, \\
q^{+}(x, t)=q^{-}(x, t) \quad \text { on } Q_{0}, \quad h^{+}(x, t)=h^{-}(x, t)=0 \quad \text { on } Q_{0}, \\
q \cdot v=u(x, t) \quad \text { on } \Sigma_{u}, \quad q \cdot v=\alpha(x) K^{*}(h)+f_{0}(x, t) \quad \text { on } \Sigma_{\alpha} .
\end{gathered}
$$

Proof. If the saturation occurs from above we will be able to represent $Q_{+}, Q_{-}$, and $Q_{0}$ as $Q_{+}=\left\{(x, t) ; 0<x_{3}<s\left(t, x_{1}, x_{2}\right)\right\}, Q_{-}=\left\{(x, t) ; s\left(t, x_{1}, x_{2}\right)<x_{3}<L\right\}$, and $Q_{0}=\left\{(x, t) ; x_{3}=\right.$ $\left.s\left(t, x_{1}, x_{2}\right)\right\}$, where $x_{3}=s\left(t, x_{1}, x_{2}\right)$ is a smooth surface.

First, in (3.1), we take $\phi$ with compact support in $Q_{-}$, and then it follows, in the sense of distributions, that

$$
-\int_{Q_{-}} C^{*}(h) \phi_{t} d x d t=\int_{Q_{-}} C_{t}^{*}(h) \phi d x d t
$$

Then

$$
\int_{Q_{-}}\left(\nabla K^{*}(h) \cdot \nabla \phi-k(h) \frac{\partial \phi}{\partial x_{3}}\right) d x d t=\int_{Q_{-}}\left(-\Delta K^{*}(h)+\frac{\partial k(h)}{\partial x_{3}}\right) \phi d x d t
$$

so that we finally get from (3.1) that

$$
\int_{Q_{-}}\left(C_{t}^{*}(h)-\Delta K^{*}(h)+\frac{\partial k(h)}{\partial x_{3}}\right) \phi d x d t=\int_{Q_{-}} f \phi d x d t, \quad \forall \phi \in C_{0}^{\infty}\left(Q_{-}\right),
$$

which implies that (3.4) is satisfied in the sense of distributions.

Similarly, if we take $\phi$ with compact support in $Q_{+}$, we get (3.5).

Now we multiply (3.4) by $\phi$, integrate it over $Q_{-}$, and add it to (3.5) multiplied by $\phi$ and integrated over $Q_{+}$. After some integrations by parts we obtain

$$
\begin{aligned}
\int_{Q_{-}}(- & \left.C^{*}(h) \phi_{t}+\nabla K^{*}(h) \cdot \nabla \phi-k(h) \frac{\partial \phi}{\partial x_{3}}\right) d x d t+\int_{\Sigma_{b}} q \cdot \nu \phi d \sigma d t \\
& +\int_{\Sigma_{\text {lat }}^{-}} q \cdot \nu \phi d \sigma d t-\int_{Q_{0}} q \cdot v^{-} \phi d \sigma d t+\int_{Q_{+}} K_{s} \nabla h \cdot \nabla \phi d x d t \\
& +\int_{\Sigma_{u}} q \cdot \nu \phi d \sigma d t+\int_{\Sigma_{\text {lat }}^{+}} q \cdot \nu \phi d \sigma d t+\int_{Q_{0}} q \cdot \nu^{+} \phi d \sigma d t \\
= & \int_{Q_{-}} f \phi d x d t+\int_{Q_{+}} f \phi d x d t+\int_{\Omega_{-}}\left(C^{*}(h) \phi\right)(x, 0) d x+\int_{\Omega_{+}}\left(C^{*}(h) \phi\right)(x, 0) d x .
\end{aligned}
$$

Here, $\Omega_{ \pm}$are the spatial domains corresponding to $Q_{ \pm}$, and $\Sigma_{\text {lat }}^{ \pm}$are the lateral boundaries corresponding to $Q_{ \pm}$, with $\Sigma_{\text {lat }}^{+} \cup \overline{\Sigma_{\text {lat }}^{-}}=\Sigma_{\text {lat }}, \Sigma_{\text {lat }}^{+} \cap \Sigma_{\text {lat }}^{-}=\varnothing$. 
Taking into account (3.1) we get

$$
\begin{gathered}
\int_{\Omega} \phi(x, 0) C^{*}\left(h_{0}\right) d x-\int_{\Sigma_{\alpha}}\left(\alpha K^{*}(h)+f_{0}\right) \phi d \sigma d t-\int_{\Sigma_{u}} u \phi d \sigma d t \\
\quad+\int_{\Sigma_{b}} q \cdot \nu \phi d \sigma d t+\int_{\Sigma_{\text {lat }}^{-}} q \cdot \nu \phi d \sigma d t-\int_{Q_{0}} q^{-} \phi d \sigma d t \\
\quad+\int_{\Sigma_{u}} q \cdot \nu \phi d \sigma d t+\int_{\Sigma_{\text {lat }}^{+}} q \cdot \nu \phi d \sigma d t+\int_{Q_{0}} q^{+} \phi d \sigma d t \\
=\int_{\Omega_{-}}\left(C^{*}(h) \phi\right)(x, 0) d x+\int_{\Omega_{+}}\left(C^{*}(h) \phi\right)(x, 0) d x,
\end{gathered}
$$

for each $\phi$ with the properties from Definition 3.1. Since $\phi$ is arbitrary, we obtain $q^{+}=$ $q^{-}$on $Q_{0}, q \cdot v=\alpha K^{*}(h)+f_{0}$ on $\Sigma_{\alpha}, q \cdot v=u$ on $\Sigma_{u}$, and $C^{*}\left(h_{0}(x)\right)=\left(C^{*}(h)\right)(x, 0)$. The condition related to zero-pressure continuity on $Q_{0}$ is implied by the assumption that $h$ is smooth and by the definition of $Q_{0}$. It must be emphasized that the three conditions on the boundary $Q_{0}$ are necessary since the free boundary is unknown.

\section{Transformed problem}

It will be convenient to work with the variable $\theta$, hence, to this end we introduce the inverse of $C^{*}$

$$
h=\left(C^{*}\right)^{-1}(\theta), \quad \theta_{\min }<\theta<\theta_{s}, \quad\left(C^{*}\right)^{-1}\left(\theta_{s}\right)=[0,+\infty)
$$

and replace all over in (2.7) $h$ by (4.1). So we get the multivalued function

$$
\beta^{*}(\theta)=K^{*}\left(\left(C^{*}\right)^{-1}(\theta)\right), \quad \theta \in\left(\theta_{\min }, \theta_{s}\right), \beta^{*}\left(\theta_{s}\right)=\left[K_{s}^{*},+\infty\right)
$$

and the conductivity expressed as a function of $\theta$

$$
K(\theta)=k\left(\left(C^{*}\right)^{-1}(\theta)\right), \quad \theta \in\left(\theta_{\min }, \theta_{s}\right]
$$

For $\theta \in\left(\theta_{\min }, \theta_{s}\right)$, we can calculate the derivative of $\beta^{*}(\theta)$, denoted by $\beta(\theta)$, which turns out to be given by

$$
\beta(\theta)=\frac{k\left(\left(C^{*}\right)^{-1}(\theta)\right)}{C\left(\left(C^{*}\right)^{-1}(\theta)\right)} \geq \frac{K_{r}}{C_{r}}=\rho,
$$

and assume that it is convex in order to respect the physical model.

Taking into account (2.2) we can extend these functions to the left of $\theta_{\min }$ by setting

$$
\beta(\theta)=\rho, \quad K(\theta)=0 \quad \text { for } \theta \leq \theta_{\min },
$$


so that we finally get

$$
\begin{aligned}
& \beta^{*}(\theta)= \begin{cases}\rho \theta, & \theta \leq \theta_{\min }, \\
K^{*}\left(\left(C^{*}\right)^{-1}(\theta)\right), & \theta_{\min }<\theta<\theta_{s}, \\
{\left[K_{s}^{*},+\infty\right),} & \theta=\theta_{s},\end{cases} \\
& K(\theta)= \begin{cases}0, & \theta \leq \theta_{\min }, \\
k\left(\left(C^{*}\right)^{-1}(\theta)\right), & \theta_{\min }<\theta \leq \theta_{s} .\end{cases}
\end{aligned}
$$

For $\theta<\theta_{s}$, we also have $\lim _{\theta>\theta_{s}} \beta^{*}(\theta)=\lim _{\theta>\theta_{s}} K^{*}\left(\left(C^{*}\right)^{-1}(\theta)\right)=\lim _{h>0} K^{*}(h)=K_{s}^{*}$. The function $\beta^{*}$ defined by (4.6) satisfies

(i) $\left(\beta^{*}(\theta)-\beta^{*}(\bar{\theta})\right)(\theta-\bar{\theta}) \geq \rho(\theta-\bar{\theta})^{2}$, for all $\theta, \bar{\theta} \in\left(-\infty, \theta_{s}\right]$,

(ii) $\lim _{\theta \rightarrow-\infty} \beta^{*}(\theta)=-\infty$.

Due to $\left(\mathrm{i}_{K}\right)$ it follows that $\theta \rightarrow K(\theta)$ is Lipschitz, that is,

$\left(\mathrm{ii}_{K}\right)|K(\theta)-K(\bar{\theta})| \leq M|\theta-\bar{\theta}|$, for all $\theta, \bar{\theta} \leq \theta_{s}$.

Condition (i) can be very easily checked for $\theta, \bar{\theta}<\theta_{s}$, or $\theta=\bar{\theta}=\theta_{s}$. If $\theta=\theta_{s}$ and $\bar{\theta}<\theta_{s}$, we have

$$
\begin{aligned}
& \left(\beta^{*}\left(\theta_{s}\right)-\beta^{*}(\bar{\theta})\right)\left(\theta_{s}-\bar{\theta}\right) \geq\left(K_{s}^{*}-\beta^{*}(\bar{\theta})\right)\left(\theta_{s}-\bar{\theta}\right) \\
& \quad=\left(\lim _{\theta>\theta_{s}} K^{*}\left(\left(C^{*}\right)^{-1}(\theta)\right)-\beta^{*}(\bar{\theta})\right)\left(\theta_{s}-\bar{\theta}\right) \geq \rho\left(\theta_{s}-\bar{\theta}\right)^{2} .
\end{aligned}
$$

With these notations the mathematical model describing the saturated-unsaturated case is reduced to the nonlinear diffusion equation

$$
\begin{gathered}
\frac{\partial \theta}{\partial t}-\Delta \beta^{*}(\theta)+\frac{\partial K(\theta)}{\partial x_{3}}=f \quad \text { in } Q, \\
\theta(x, 0)=\theta_{0}(x) \quad \text { in } \Omega, \\
\left(K(\theta) i_{3}-\nabla \beta^{*}(\theta)\right) \cdot v=u \text { on } \Sigma_{u}, \\
\left(K(\theta) i_{3}-\nabla \beta^{*}(\theta)\right) \cdot v=\alpha \beta^{*}(\theta)+f_{0} \text { on } \Sigma_{\alpha} .
\end{gathered}
$$

From this point we may further have two approaches. If we are interested in the study of the occurrence of saturation in a porous medium and in the advance of the free boundary between the saturated and unsaturated domains, we will analyze model (4.9) for $\theta \leq \theta_{s}$, with $\beta^{*}(\theta)$ being the multivalued operator given by (4.6), with properties (i), (ii), and $K$ being the continuous function given by (4.7) satisfying ( $\mathrm{ii}_{K}$ ).

But in soil sciences, the interest is often in the unsaturated flow only, so that model (4.9) is studied for $\theta<\theta_{s}$, with $\beta^{*}$ being the function defined strictly in this domain, satisfying (i) but with blowing up at $\theta_{s}$. This situation has already been considered in [6] and for a stratified soil in [5].

The theoretical results presented in the work can be applied to the parametric hydraulic model of Broadbridge and White introduced in [14], which in our notations read as follows:

$$
K(\theta)=\frac{(c-1) \theta^{2}}{c-\theta}, \quad \beta(\theta)=\frac{c(c-1)}{(c-\theta)^{2}}
$$


Here, $c \in(1, \infty)$ is a parameter that indicates the degree of nonlinearity of the medium, that is, when $c$ is close to $1, \theta \rightarrow 1$ and the soil is strongly nonlinear. Moreover, $K(0)=0$ and $\beta(0)=(c-1) / c>0$, corresponding to the conditions imposed above for the case $\theta_{\min }=0$.

Functional framework. We consider the space $V=H^{1}(\Omega)$, with the norm defined by

$$
\|\psi\|_{V}=\left(\int_{\Omega}|\nabla \psi|^{2} d x+\int_{\Gamma_{\alpha}} \alpha(x)|\psi|^{2} d \sigma\right)^{1 / 2}
$$

which is equivalent to the usual norm on $H^{1}(\Omega)$. Let $V^{\prime}=\left(H^{1}(\Omega)\right)^{\prime}$ be the dual of $V$. It is convenient to endow the dual $V^{\prime}$ with the scalar product

$$
\langle\theta, \bar{\theta}\rangle_{V^{\prime}}=(\theta, \psi), \quad \forall \theta, \bar{\theta} \in V^{\prime},
$$

where $\psi \in V$ satisfies the boundary value problem

$$
-\Delta \psi=\bar{\theta}, \quad \frac{\partial \psi}{\partial \nu}+\alpha \psi=0 \quad \text { on } \Gamma_{\alpha}, \quad \frac{\partial \psi}{\partial \nu}=0 \quad \text { on } \Gamma_{u},
$$

meaning that

$$
\int_{\Omega} \nabla \psi \cdot \nabla \phi d x+\int_{\Gamma_{\alpha}} \alpha \psi \phi d \sigma=\bar{\theta}(\phi), \quad \forall \phi \in V
$$

$\left(\partial / \partial \nu\right.$ is the normal derivative). Obviously $\|\psi\|_{V}=\|\bar{\theta}\|_{V^{\prime}}$. We set

$$
D(A)=\left\{\theta \in L^{2}(\Omega) ; \exists \eta \in V \text { and } \eta(x) \in \beta^{*}(\theta(x)) \text { a.e. } x \in \Omega\right\}
$$

and we define the multivalued operator $A: D(A) \subset V^{\prime} \rightarrow V^{\prime}$ by

$$
(A \theta, \psi)=\int_{\Omega}\left(\nabla \eta \cdot \nabla \psi-K(\theta) \frac{\partial \psi}{\partial x_{3}}\right) d x+\int_{\Gamma_{\alpha}} \alpha \eta \psi d \sigma, \quad \forall \psi \in V .
$$

We still define $B \in L\left(L^{2}\left(\Gamma_{u}\right) ; V^{\prime}\right)$ and $f_{\Gamma} \in L^{2}\left(0, T ; V^{\prime}\right)$ by

$$
\begin{aligned}
B u(\psi)=-\int_{\Gamma_{u}} u \psi d \sigma, & \forall \psi \in V, \\
f_{\Gamma}(t)(\psi)=-\int_{\Gamma_{\alpha}} f_{0} \psi d \sigma, & \forall \psi \in V,
\end{aligned}
$$

and so we are led to the Cauchy problem

$$
\begin{array}{cc}
\frac{d \theta}{d t}+A \theta \ni f+B u+f_{\Gamma} & \text { a.e. } t \in(0, T) \\
\theta(0)=\theta_{0}(x) & \text { in } \Omega .
\end{array}
$$


Equation (4.21) can still be written equivalently in the form

$$
\begin{aligned}
& \int_{\Omega}(\left.\frac{\partial \theta}{\partial t} \psi+\nabla \eta \cdot \nabla \psi-K(\theta) \frac{\partial \psi}{\partial x_{3}}\right) d x \\
& \quad=\int_{\Omega} f \psi d x-\int_{\Gamma_{\alpha}}\left(\alpha \eta+f_{0}\right) \psi d \sigma-\int_{\Gamma_{u}} u \psi d \sigma, \quad \forall \psi \in V, t \in(0, T),
\end{aligned}
$$

for some $\eta \in \beta^{*}(\theta)$. One can show that if $\theta$ is a strong solution to the Cauchy problem (4.21)-(4.22), then it satisfies (4.9)-(4.12) (see [6]).

4.1. The approximating problem. We now assume that $\beta^{*}$ is defined to be of class $C^{3}$ on $\theta<\theta_{s}$ and such that it preserves the condition that $\lim _{\theta \rightarrow-\infty} \beta^{*}(\theta)=-\infty$. That means we have imposed that the functions $C$ and $k$ should be of class $C^{2}$ on $h<0$. Although this assumption is concordant with the physical model and could be imposed without any loss of generality, it is not absolutely necessary since we can approximate a non sufficiently smooth function by sequences of functions having the necessary smoothness, and pass then to the limit in the approximating equations.

So, in order to prove the existence and uniqueness results, we approximate $\beta^{*}$ by the continuous function

$$
\beta_{\varepsilon}^{*}(\theta)= \begin{cases}\beta^{*}(\theta), & \theta<\theta_{s} \\ K_{s}^{*}+\frac{\theta-\theta_{s}}{\varepsilon}, & \theta \geq \theta_{s}\end{cases}
$$

for each $\varepsilon>0$, so $\beta_{\varepsilon}^{*}(\theta)$ satisfies properties (i), (ii), and

(iii) $\lim _{\theta \rightarrow \infty} \beta_{\varepsilon}^{*}(\theta)=+\infty$.

Another way to approximate $\beta^{*}$ is the following, for which $\beta_{\varepsilon}^{*}$ is continuous and differentiable except for $\theta_{s}-\varepsilon$ :

$$
\beta_{\varepsilon}^{*}(\theta)= \begin{cases}\beta^{*}(\theta), & \theta<\theta_{s}-\varepsilon, \\ \beta^{*}\left(\theta_{s}-\varepsilon\right)+\frac{K_{s}^{*}-\beta^{*}\left(\theta_{s}-\varepsilon\right)}{\varepsilon}\left[\theta-\left(\theta_{s}-\varepsilon\right)\right], & \theta \geq \theta_{s}-\varepsilon .\end{cases}
$$

Hence we have to study the approximating problem

$$
\begin{gathered}
\frac{d \theta_{\varepsilon}}{d t}+A_{\varepsilon} \theta_{\varepsilon}=f+B u+f_{\Gamma} \quad \text { a.e. } t \in(0, T), \\
\theta_{\varepsilon}(0)=\theta_{0}(x) \quad \text { in } \Omega,
\end{gathered}
$$

where $A_{\varepsilon}: D\left(A_{\varepsilon}\right) \subset V^{\prime} \rightarrow V^{\prime}$ is a single-valued operator defined by

$$
\left(A_{\varepsilon} \theta, \psi\right)=\int_{\Omega}\left(\nabla \beta_{\varepsilon}^{*}(\theta) \cdot \nabla \psi-K(\theta) \frac{\partial \psi}{\partial x_{3}}\right) d x+\int_{\Gamma_{\alpha}} \alpha \beta_{\varepsilon}^{*}(\theta) \psi d \sigma, \quad \forall \psi \in V,
$$


with the domain

$$
D\left(A_{\varepsilon}\right)=\left\{\theta \in L^{2}(\Omega) ; \beta_{\varepsilon}^{*}(\theta) \in V\right\}
$$

4.2. Main results. The results presented below refer to the properties of the operator $A_{\varepsilon}$ (Proposition 4.1), existence, uniqueness, and regularity of the solution to the approximating problem (Proposition 4.2 and Theorem 4.3), existence and uniqueness of the solution to the exact Cauchy problem (Theorem 4.4), some properties of the solution (Corollaries 4.5 and 4.6), and the existence of the weak solution (Corollary 4.8). In all these proofs we will use the approximation (4.24).

Proposition 4.1. Under the conditions (i)-(iii), ( $\left(i_{K}\right)$ the operator $A_{\varepsilon}$ is quasi m-accretive in $V^{\prime}$, meaning that

$$
\left\langle\left(\lambda I+A_{\varepsilon}\right) \theta-\left(\lambda I+A_{\varepsilon}\right) \bar{\theta}, \theta-\bar{\theta}\right\rangle_{V^{\prime}} \geq 0
$$

for $\lambda>0$ large enough, and

$$
R\left(\lambda I+A_{\varepsilon}\right)=V^{\prime}
$$

for some $\lambda$ sufficiently large.

Since the proofs of Propositions 4.1 and 4.2 and Theorem 4.3 are essentially the same as those of [6, Proposition 1, Theorem 1, and Corollary 1, (a)], they will be omitted, and for their details, we refer the reader to this work.

Let $j_{\varepsilon}: \mathbb{R} \rightarrow(-\infty, \infty)$ be defined by

$$
j_{\mathcal{\varepsilon}}(r)=\int_{0}^{r} \beta_{\varepsilon}^{*}(\xi) d \xi
$$

By Proposition 4.1 and by standard existence results for nonlinear accretive differential equations (see [4]), we obtain the following proposition.

Proposition 4.2. Let

$$
\begin{gathered}
f \in W^{1,1}\left(0, T ; V^{\prime}\right), \quad f_{0} \in W^{1,1}\left(0, T ; L^{2}\left(\Gamma_{\alpha}\right)\right), \quad u \in W^{1,1}\left(0, T ; L^{2}\left(\Gamma_{u}\right)\right), \\
\theta_{0} \in D\left(A_{\varepsilon}\right)
\end{gathered}
$$

and assume that conditions (i)-(iii), ( $\left.i_{K}\right)$ hold. Then, for each $\varepsilon$, there exists a unique strong solution $\theta_{\varepsilon}$ to problem (4.26)-(4.27) such that

$$
\begin{gathered}
\theta_{\varepsilon} \in L^{\infty}\left(0, T ; D\left(A_{\varepsilon}\right)\right) \cap W^{1, \infty}\left(0, T ; V^{\prime}\right), \\
\beta_{\varepsilon}^{*}\left(\theta_{\varepsilon}\right) \in L^{\infty}(0, T ; V), \quad \theta_{\varepsilon} \in L^{\infty}(0, T ; V), \\
j_{\varepsilon}\left(\theta_{\varepsilon}\right) \in L^{\infty}\left(0, T ; L^{1}(\Omega)\right) .
\end{gathered}
$$


Moreover, the solution satisfies the estimates

$$
\begin{gathered}
\left\|\theta_{\varepsilon}(t)\right\|_{V^{\prime}}^{2}+\int_{0}^{t}\left\|\theta_{\varepsilon}(\tau)\right\|^{2} d \tau \\
\leq \gamma_{1}\left(\alpha_{m}\right)\left(\left\|\theta_{0}\right\|_{V^{\prime}}^{2}+\int_{0}^{T}\|f(\tau)\|_{V^{\prime}}^{2} d \tau+\int_{0}^{T}\|u(\tau)\|_{L^{2}\left(\Gamma_{u}\right)}^{2} d \tau+\int_{0}^{T}\left\|f_{0}(\tau)\right\|_{L^{2}\left(\Gamma_{\alpha}\right)}^{2} d \tau\right) \\
\left\|\theta_{\varepsilon}(t)\right\|^{2} \leq \int_{\Omega} j_{\mathcal{\varepsilon}}\left(\theta_{\varepsilon}(t)\right) d x+\int_{0}^{t}\left\|\frac{d \theta_{\varepsilon}}{d \tau}(\tau)\right\|_{V^{\prime}}^{2} d \tau+\int_{0}^{t}\left\|\beta_{\varepsilon}^{*}\left(\theta_{\varepsilon}(\tau)\right)\right\|_{V}^{2} d \tau \\
\leq \gamma_{2}\left(\alpha_{m}\right)\left(\int_{\Omega} j_{\mathcal{\varepsilon}}\left(\theta_{0}\right) d x+\int_{0}^{T}\|f(\tau)\|_{V^{\prime}}^{2} d \tau\right. \\
\left.\quad+\int_{0}^{T}\|u(\tau)\|_{L^{2}\left(\Gamma_{u}\right)}^{2} d \tau+\int_{0}^{T}\left\|f_{0}(\tau)\right\|_{L^{2}\left(\Gamma_{\alpha}\right)}^{2} d \tau\right) .
\end{gathered}
$$

In the above estimates, $\alpha_{m}=\min _{x \in \Gamma_{\alpha}} \alpha(x), \gamma_{1}\left(\alpha_{m}\right)=O\left(1 / \alpha_{m}\right)$, and $\gamma_{2}\left(\alpha_{m}\right)=O\left(1 / \alpha_{m}\right)$. Also we notice that it follows that $K\left(\theta_{\varepsilon}\right) \in L^{\infty}(0, T ; V)$.

Let now $j: \mathbb{R} \rightarrow(-\infty, \infty]$ be defined by

$$
j(r)= \begin{cases}\int_{0}^{r} \beta^{*}(\xi) d \xi, & r \leq \theta_{s} \\ +\infty, & r>\theta_{s}\end{cases}
$$

where, by convention, $\beta^{*}\left(\theta_{s}\right)=\lim _{\substack{\xi \rightarrow \theta_{s} \\ \xi<\theta_{s}}} \beta^{*}\left(\theta_{s}\right)=K_{s}^{*}$.

Then (see [11]) $j$ is a proper, convex, and lower semicontinuous function on $\mathbb{R}$ and

$$
\partial j(r)=\beta^{*}(r)
$$

In particular, $\beta^{*}$ is a maximal monotone operator from $\mathbb{R}$ to $\mathbb{R}$.

Denote $M_{\theta_{s}}=\left\{\theta \in L^{2}(\Omega) ; \theta \leq \theta_{s}\right.$ a.e. on $\left.\Omega\right\}$ and $M_{j}=\left\{\theta \in L^{2}(\Omega) ; j(\theta) \in L^{1}(\Omega)\right\}$. Then $M_{\theta_{s}} \subset \overline{M_{j}}$.

Theorem 4.3. Let

$$
f \in L^{2}\left(0, T ; V^{\prime}\right), \quad u \in L^{2}\left(0, T ; L^{2}\left(\Gamma_{u}\right)\right), \quad f_{0} \in L^{2}\left(0, T ; L^{2}\left(\Gamma_{\alpha}\right)\right), \quad \theta_{0} \in M_{\theta_{s}} \text {. }
$$

Then, problem (4.26)-(4.27) has, for each $\varepsilon>0$, a unique solution that satisfies estimates (4.35)-(4.36) and

$$
\theta_{\varepsilon} \in W^{1,2}\left(0, T ; V^{\prime}\right), \quad \beta_{\varepsilon}^{*}(\theta) \in L^{2}(0, T ; V), \quad \theta_{\varepsilon} \in L^{2}(0, T ; V) .
$$


Proof. If $\theta_{0} \leq \theta_{s}$, we have

$$
j_{\varepsilon}\left(\theta_{0}\right)=\int_{0}^{\theta_{0}} \beta_{\varepsilon}^{*}(\theta) d \theta \leq \int_{0}^{\theta_{s}} \beta_{\varepsilon}^{*}(\theta) d \theta \leq \int_{0}^{\theta_{s}} \beta^{*}(\theta) d \theta=j\left(\theta_{s}\right) \leq K_{s}^{*} \theta_{s} .
$$

This means that $\theta_{0} \in D\left(\varphi_{\varepsilon}\right)$, where $\varphi_{\varepsilon}(\theta)=\int_{\Omega} j_{\varepsilon}(\theta) d x$ and $\overline{D\left(\varphi_{\varepsilon}\right)}=\overline{D\left(A_{\varepsilon}\right)}$.

Due to density arguments, let $\left\{f_{n}\right\},\left\{u_{n}\right\}$, and $\left\{f_{n}^{0}\right\}$ be three sequences such that

$$
\begin{aligned}
f_{n} \in W^{1,1}\left(0, T ; V^{\prime}\right), & f_{n} \longrightarrow f \text { in } L^{2}\left(0, T ; V^{\prime}\right), \\
u_{n} \in W^{1,1}\left(0, T ; L^{2}\left(\Gamma_{u}\right)\right), & u_{n} \longrightarrow u \text { in } L^{2}\left(0, T ; L^{2}\left(\Gamma_{u}\right)\right), \\
f_{n}^{0} \in W^{1,1}\left(0, T ; L^{2}\left(\Gamma_{\alpha}\right)\right), & f_{n}^{0} \longrightarrow f_{0} \text { in } L^{2}\left(0, T ; L^{2}\left(\Gamma_{\alpha}\right)\right),
\end{aligned}
$$

and let $\theta_{0} \in L^{2}(\Omega), \theta_{0} \leq \theta_{s}$. Then there exists $\left\{\left(\theta_{0}\right)_{n}\right\} \subset D\left(A_{\varepsilon}\right)$ such that $\left(\theta_{0}\right)_{n} \rightarrow \theta_{0}$ in $V^{\prime}$. Then, for each $\varepsilon>0$, there is a unique solution $\left(\theta_{\varepsilon}\right)_{n}$ to the approximating problem

$$
\begin{gathered}
\frac{d\left(\theta_{\varepsilon}\right)_{n}}{d t}+A_{\varepsilon}\left(\theta_{\varepsilon}\right)_{n}=f_{n}+B u_{n}+f_{n}^{\Gamma} \quad \text { a.e. } t \in(0, T), \\
\left(\theta_{\varepsilon}\right)_{n}(0)=\left(\theta_{0}\right)_{n}(x) \quad \text { in } \Omega,
\end{gathered}
$$

that satisfies (4.34)-(4.36) and

$$
\begin{aligned}
\left\|\left(\theta_{\varepsilon}\right)_{n}(t)-\left(\theta_{\varepsilon}\right)_{m}(t)\right\|_{V^{\prime}}^{2}+\int_{0}^{t}\left\|\left(\theta_{\varepsilon}\right)_{n}(\tau)-\left(\theta_{\varepsilon}\right)_{m}(t)\right\|^{2} d \tau \\
\leq \gamma_{3}\left(\alpha_{m}\right)\left(\left\|\left(\theta_{0}\right)_{n}-\left(\theta_{0}\right)_{m}\right\|_{V^{\prime}}^{2}+\int_{0}^{T}\left\|f_{n}(\tau)-f_{m}(\tau)\right\|_{V^{\prime}}^{2} d \tau\right. \\
\left.\quad+\int_{0}^{T}\left\|u_{n}(\tau)-u_{m}(\tau)\right\|_{L^{2}\left(\Gamma_{u}\right)}^{2} d \tau+\int_{0}^{T}\left\|f_{n}^{0}(\tau)-f_{m}^{0}(\tau)\right\|_{L^{2}\left(\Gamma_{\alpha}\right)}^{2} d \tau\right) .
\end{aligned}
$$

By passing to the limit as $n \rightarrow 0$, we obtain the results of Theorem 4.3, as claimed. Moreover, we deduce that $K\left(\theta_{\varepsilon}\right) \in L^{2}(0, T ; V)$.

In Theorem 4.3, letting $\varepsilon$ tend to 0 , we obtain the following existence result.

Theorem 4.4. Let $f, u, f_{0}$, and $\theta_{0}$ satisfy (4.39). Then there exists a unique solution $\theta$ to the exact problem (4.21)-(4.22) with the following properties:

$$
\begin{gathered}
\theta \in L^{2}(0, T ; V) \cap W^{1,2}\left(0, T ; V^{\prime}\right), \quad \beta^{*}(\theta) \in L^{2}(0, T ; V), \\
K(\theta) \in L^{2}(0, T ; V), \quad j(\theta) \in L^{\infty}\left(0, T ; L^{1}(\Omega)\right) .
\end{gathered}
$$

Proof. Assume that (4.39) holds. Then the approximating problem (4.26)-(4.27) has a strong solution $\theta_{\varepsilon}$ satisfying (4.40), (4.35), and (4.36).

Since for $\theta_{0} \leq \theta_{s}$ we have $j_{\varepsilon}\left(\theta_{0}\right) \leq K_{s}^{*} \theta_{s}$, the right-hand side term in (4.36) is bounded independently of $\varepsilon$. Hence, from (4.35) and (4.36), we deduce that $\left\{\theta_{\varepsilon}\right\}$ lies in a bounded subset of $L^{\infty}\left(0, T ; L^{2}(\Omega)\right),\left\{d \theta_{\varepsilon} / d t\right\}$ lies in a bounded subset of $L^{2}\left(0, T ; V^{\prime}\right)$, and $\left\{\beta_{\varepsilon}^{*}\left(\theta_{\varepsilon}\right)\right\}$ is in a bounded subset of $L^{2}(0, T ; V)$. Using (ii), we get that $\left(\beta_{\varepsilon}^{*}\right)^{-1}$ is Lipschitz, and hence $\left\{\theta_{\varepsilon}\right\}$ is in a bounded subset of $L^{2}(0, T ; V)$ too. 
So, from the boundedness of the sequences previously mentioned, we conclude that there exists a subsequence (that will be denoted $\theta_{\varepsilon}$ too) such that

$$
\begin{gathered}
\theta_{\varepsilon} \longrightarrow \theta \quad \text { weakly in } L^{2}(0, T ; V) \text { and weak star in } L^{\infty}\left(0, T ; L^{2}(\Omega)\right), \\
\frac{d \theta_{\varepsilon}}{d t} \longrightarrow \frac{d \theta}{d t} \quad \text { weakly in } L^{2}\left(0, T ; V^{\prime}\right) .
\end{gathered}
$$

Since $V=H_{0}^{1}(\Omega)$ is compactly embedded in $H=L^{2}(\Omega)$ by Lions-Aubin compactness theorem (see [10]), we conclude that $\left\{\theta_{\varepsilon}\right\}$ is compact in $L^{2}\left(0, T ; L^{2}(\Omega)\right.$ ), that is,

$$
\begin{aligned}
& \theta_{\varepsilon} \longrightarrow \theta \quad \text { strongly in } L^{2}\left(0, T ; L^{2}(\Omega)\right), \text { as } \varepsilon \longrightarrow 0 \\
& \theta_{\varepsilon}(t) \longrightarrow \theta(t) \quad \text { weakly in } V^{\prime}, \text { for each } t \in[0, T]
\end{aligned}
$$

We obtain also that $\theta(0)=\theta_{0}$. Since $\theta \rightarrow K(\theta)$ is continuous from $L^{2}(0, T ; V)$ to $L^{2}(0, T$; $\left.L^{2}(\Omega)\right)$, it follows that

$$
K\left(\theta_{\varepsilon}\right) \longrightarrow K(\theta) \quad \text { strongly in } L^{2}\left(0, T ; L^{2}(\Omega)\right) \text {, as } \varepsilon \longrightarrow 0 \text {. }
$$

From (4.36) we may assume that

$$
\beta_{\varepsilon}^{*}\left(\theta_{\varepsilon}\right) \longrightarrow \eta \quad \text { weakly in } L^{2}(0, T ; V) .
$$

Moreover, by the trace theorem, it follows that $\beta_{\varepsilon}^{*}\left(\theta_{\varepsilon}\right) \rightarrow \eta$ strongly in $L^{2}(\Sigma)$.

We will prove that $\eta \in \beta^{*}(\theta)$ a.e. on $\Omega$. Indeed, from the obvious inequality

$$
\int_{\Omega} j_{\varepsilon}(\theta) d x \leq \int_{\Omega} j_{\varepsilon}\left(\theta_{\varepsilon}\right) d x+\int_{\Omega} \beta_{\varepsilon}^{*}(\theta)\left(\theta-\theta_{\varepsilon}\right) d x, \quad \forall \theta \in L^{2}(\Omega),
$$

we have by passing to the limit as $\varepsilon \rightarrow 0$ that

$$
\liminf _{\varepsilon \rightarrow 0} \int_{\Omega} j_{\varepsilon}(\theta) d x \leq \liminf _{\varepsilon \rightarrow 0} \int_{\Omega} j_{\varepsilon}\left(\theta_{\varepsilon}\right) d x
$$

Since $j_{\mathcal{\varepsilon}}(\theta)>0$, we have by Fatou's lemma that

$$
\int_{\Omega} j(\theta) d x \leq \liminf _{\varepsilon \rightarrow 0} \int_{\Omega} j_{\varepsilon}(\theta) d x
$$

On the other hand, we have

$$
\int_{Q} \beta_{\varepsilon}^{*}\left(\theta_{\varepsilon}\right)\left(\theta_{\varepsilon}-z\right) d x d t \geq \int_{Q} j_{\varepsilon}\left(\theta_{\varepsilon}\right) d x d t-\int_{Q} j_{\varepsilon}(z) d x d t
$$


for all $z \in L^{2}(Q), z \leq \theta_{s}$ a.e. on $Q$. Passing to the limit as $\varepsilon \rightarrow 0$, we obtain that

$$
\int_{Q} \eta(\theta-z) d x \geq \int_{Q} j(\theta) d x-\int_{Q} j(z) d x, \quad \forall z \in L^{2}(Q), z \leq \theta_{s} \text { a.e. on } Q \text {. }
$$

This means that $\eta \in \beta^{*}(\theta)$ a.e. on $\Omega$.

In the above calculations we used the fact that $j_{\varepsilon}(z) \rightarrow j(z)$, for all $z \in L^{2}(\Omega)$, which is an obvious assertion for $z \leq \theta_{s}$. Now, if $z>\theta_{s}$, we obtain

$$
j_{\varepsilon}(z)=\int_{0}^{z} \beta_{\varepsilon}^{*}(r) d r=K_{s}^{*} z+\frac{\left(z-\theta_{s}\right)^{2}-\theta_{s}^{2}}{2 \varepsilon} \longrightarrow+\infty=j(z) \quad \text { as } \varepsilon \longrightarrow 0 .
$$

By (4.26), we obtain

$$
\begin{aligned}
\int_{Q}\left(\frac{\partial \theta_{\varepsilon}}{\partial t} \psi+\nabla \beta_{\varepsilon}^{*}(\theta) \cdot \nabla \psi-K\left(\theta_{\varepsilon}\right) \frac{\partial \psi}{\partial x_{3}}\right) d x d t \\
\quad=\int_{Q} f \psi d x d t-\int_{\Sigma_{\alpha}}\left(\alpha \beta_{\varepsilon}^{*}(\theta)+f_{0}\right) \psi d \sigma d t-\int_{\Sigma_{u}} u \psi d \sigma d t, \quad \forall \psi \in V .
\end{aligned}
$$

Passing to the limit as $\varepsilon \rightarrow 0$, we get

$$
\begin{aligned}
& \int_{Q}(\left.\frac{\partial \theta}{\partial t} \psi+\nabla \eta \cdot \nabla \psi-K(\theta) \frac{\partial \psi}{\partial x_{3}}\right) d x d t \\
& \quad=\int_{Q} f \psi d x d t-\int_{\Sigma_{\alpha}}\left(\alpha \eta+f_{0}\right) \psi d \sigma d t-\int_{\Sigma_{u}} u \psi d \sigma d t, \quad \forall \psi \in V,
\end{aligned}
$$

so $\theta$ is a solution to (4.21)-(4.22). The uniqueness follows from the estimate of the difference between the two solutions.

Corollary 4.5. Let $f, u, f_{0}$, and $\theta_{0}$ satisfy (4.39). Then, the solution $\theta$ to (4.21)-(4.22) has the property that $\theta(x, t) \leq \theta_{s}$, a.e. in $Q$.

Proof. By (4.36), we have that

$$
\int_{0}^{t}\left\|\beta_{\varepsilon}^{*}\left(\theta_{\varepsilon}(\tau)\right)\right\|^{2} d \tau \leq \int_{0}^{t}\left\|\beta_{\varepsilon}^{*}\left(\theta_{\varepsilon}(\tau)\right)\right\|_{V}^{2} d \tau \leq c_{0}
$$

If we denote by $\chi_{\varepsilon}^{+}(x, t)$ the characteristic function of $Q_{\varepsilon}^{+}$and denote $Q_{\varepsilon}^{-}=\{(x, t) \in Q$; $\left.\theta_{\varepsilon}(x, t) \leq \theta_{s}\right\}$ and $Q_{\varepsilon}^{+}=\left\{(x, t) \in Q ; \theta_{\varepsilon}(x, t)>\theta_{s}\right\}$, we have

$$
\int_{0}^{t}\left\|\beta_{\varepsilon}^{*}\left(\theta_{\varepsilon}(\tau)\right)\right\|^{2} d \tau=\int_{Q_{\varepsilon}^{-}}\left(\beta_{\varepsilon}^{*}\left(\theta_{\varepsilon}\right)\right)^{2} d x d t+\int_{Q_{\varepsilon}^{+}}\left(\beta_{\varepsilon}^{*}\left(\theta_{\varepsilon}\right)\right)^{2} d x d t \leq c_{0}
$$

wherefrom, using that $\beta_{\varepsilon}^{*}\left(\theta_{\varepsilon}\right) \leq K_{s}^{*}$ on $Q_{\varepsilon}^{-}$, we get

$$
\int_{Q} \chi_{\varepsilon}^{+}(x, t)\left(K_{s}^{*}+\frac{\theta_{\varepsilon}-\theta_{s}}{\varepsilon}\right)^{2} d x d t \leq c_{1} .
$$


This implies after some calculations that

$$
\int_{Q} \chi_{\varepsilon}^{+}(x, t)\left(\theta_{\varepsilon}-\theta_{s}\right)^{2} d x d t \leq c_{2} \varepsilon^{2}
$$

with $c_{0}, c_{1}$, and $c_{2}$ some constants. But

$$
\liminf _{\varepsilon \rightarrow 0} \chi_{\varepsilon}^{+}(x, t) \geq \chi^{+}(x, t) \quad \text { a.e. on } Q,
$$

hence

$$
\liminf _{\varepsilon \rightarrow 0} \chi_{\varepsilon}^{+}(x, t)\left(\theta_{\varepsilon}-\theta_{s}\right)^{2} \geq \chi^{+}(x, t)\left(\theta-\theta_{s}\right)^{2} \quad \text { a.e. on } Q \text {. }
$$

By Fatou's lemma, we have

$$
\int_{Q} \chi^{+}(x, t)\left(\theta-\theta_{s}\right)^{2} d x d t \leq \liminf _{\varepsilon \rightarrow 0} \int_{Q} \chi_{\varepsilon}^{+}(x, t)\left(\theta_{\varepsilon}-\theta_{s}\right)^{2} d x d t=0,
$$

where $\chi^{+}(x, t)$ is the characteristic function of $Q^{+}=\left\{(x, t) \in Q ; \theta(x, t)>\theta_{s}\right\}$. This yields that $\chi^{+}(x, t)=0$, meaning that $\theta(x, t) \leq \theta_{s}$, a.e. $(x, t) \in Q$.

Concerning $\theta_{r}$, the relationship $\theta(x, t) \geq \theta_{r}$ does not generally hold. Actually that would have been expected, because the fact that the moisture in a soil does not go under the residual value $\theta_{r}$ is determined by factors that have not been taken into account in our model. But instead of this, we can prove under some hypotheses that $\theta(x, t) \geq \theta_{\min }$ a.e. If, in particular, $\theta_{\min }=0$, meaning that the relation $\theta_{r}=C_{r}$ takes place, then $\theta(x, t) \geq 0$.

Corollary 4.6. Let $f, u, f_{0}$, and $\theta_{0}$ satisfy (4.39) and assume that

$$
\theta_{0} \geq \theta_{\min } \quad \text { in } \Omega, \quad f \geq 0 \quad \text { in } Q, \quad u \leq 0 \quad \text { on } \Sigma_{u}, \quad f_{0} \leq 0 \quad \text { on } \Sigma_{\alpha} .
$$

Then, the solution $\theta$ to (4.21)-(4.22) is in $L^{\infty}(Q)$ and satisfies $\theta(x, t) \geq \theta_{\min }$ a.e. in $Q$.

Proof. Assume that $\theta_{0} \geq \theta_{\min }$, meaning that the negative part $\left(\theta_{0}-\theta_{\min }\right)^{-}=0$. We have to show that $\left(\theta_{\varepsilon}-\theta_{\min }\right)^{-}=0$ too. We multiply $(4.26)$ by $\left(\theta_{\varepsilon}-\theta_{\min }\right)^{-}$and integrate over $\Omega \times(0, t)$. Using Stampacchia's lemma (see, e.g., [4]),

$$
\nabla \theta^{-}= \begin{cases}-\nabla \theta, & \text { a.e. on } \theta<0 \\ 0, & \text { a.e on } \theta \geq 0\end{cases}
$$

we have since $\theta_{\min }$ is a constant

$$
\begin{aligned}
\int_{0}^{t} \int_{\Omega}\{ & \left.-\frac{1}{2} \frac{d}{d \tau}\left[\left(\theta_{\varepsilon}-\theta_{\min }\right)^{-}\right]^{2}+\nabla \beta_{\varepsilon}^{*}\left(\theta_{\varepsilon}\right) \cdot \nabla\left(\theta_{\varepsilon}-\theta_{\min }\right)^{-}-K\left(\theta_{\varepsilon}\right) \frac{\partial\left(\theta_{\varepsilon}-\theta_{\min }\right)^{-}}{\partial x_{3}}\right\} d x d \tau \\
= & \int_{0}^{t} \int_{\Omega} f\left(\theta_{\varepsilon}-\theta_{\min }\right)^{-} d x d \tau-\int_{0}^{t} \int_{\Gamma_{\alpha}}\left(\alpha \beta_{\varepsilon}^{*}\left(\theta_{\varepsilon}\right)+f_{0}\right)\left(\theta_{\varepsilon}-\theta_{\min }\right)^{-} d \sigma d \tau \\
& -\int_{0}^{t} \int_{\Gamma_{u}} u\left(\theta_{\varepsilon}-\theta_{\min }\right)^{-} d \sigma d \tau .
\end{aligned}
$$


After integrating the first term on the left-hand side with respect to $t$, we get

$$
\begin{aligned}
-\frac{1}{2} \int_{\Omega} & {\left[\left(\theta_{\varepsilon}(t)-\theta_{\min }\right)^{-}\right]^{2} d x-\int_{0}^{t} \int_{\Omega} \beta_{\varepsilon}\left(\theta_{\varepsilon}\right)\left|\nabla\left(\theta_{\varepsilon}-\theta_{\min }\right)^{-}\right|^{2} d x d \tau } \\
& -\int_{0}^{t} \int_{\Omega} K\left(\theta_{\varepsilon}\right) \frac{\partial\left(\theta_{\varepsilon}-\theta_{\min }\right)^{-}}{\partial x_{3}} d x d \tau+\int_{0}^{t} \int_{\Gamma_{\alpha}} \alpha \beta_{\varepsilon}^{*}\left(\theta_{\varepsilon}\right)\left(\theta_{\varepsilon}-\theta_{\min }\right)^{-} d \sigma d \tau \\
= & \int_{0}^{t} \int_{\Omega} f\left(\theta_{\varepsilon}-\theta_{\min }\right)^{-} d x d \tau \\
& -\int_{0}^{t} \int_{\Gamma_{\alpha}} f_{0}\left(\theta_{\varepsilon}-\theta_{\min }\right)^{-} d \sigma d \tau-\int_{0}^{t} \int_{\Gamma_{u}} u\left(\theta_{\varepsilon}-\theta_{\min }\right)^{-} d \sigma d \tau .
\end{aligned}
$$

We took into account that $\theta \theta^{-}=-\left(\theta^{-}\right)^{2}$. But, since $\beta_{\varepsilon}^{*}\left(\theta_{\varepsilon}\right)=\int_{\theta_{\min }}^{\theta_{\varepsilon}} \beta_{\varepsilon}(\xi) d \xi \geq \rho\left(\theta_{\varepsilon}-\theta_{\min }\right)$, we have

$$
-\rho \int_{0}^{t} \int_{\Gamma_{\alpha}} \alpha_{m}\left[\left(\theta_{\varepsilon}-\theta_{\min }\right)^{-}\right]^{2} d \sigma d \tau \leq \int_{0}^{t} \int_{\Gamma_{\alpha}} \alpha \beta^{*}\left(\theta_{\varepsilon}\right)\left(\theta_{\varepsilon}-\theta_{\min }\right)^{-} d \sigma d \tau .
$$

Therefore, setting $\rho_{\alpha}=\min \left(\rho, \alpha_{m} \rho\right)$, we get

$$
\begin{aligned}
\frac{1}{2} \int_{\Omega}[ & \left.\left(\theta_{\varepsilon}(t)-\theta_{\min }\right)^{-}\right]^{2} d x+\rho_{\alpha} \int_{0}^{t}\left\|\left(\theta_{\varepsilon}(t)-\theta_{\min }\right)^{-}\right\|_{V}^{2} d \tau \\
= & -\int_{0}^{t} \int_{\Omega} K\left(\theta_{\varepsilon}\right) \frac{\partial\left(\theta_{\varepsilon}-\theta_{\min }\right)^{-}}{\partial x_{3}} d x d t-\int_{0}^{t} \int_{\Omega} f\left(\theta_{\varepsilon}-\theta_{\min }\right)^{-} d x d \tau \\
& +\int_{0}^{t} \int_{\Gamma_{\alpha}} f_{0}\left(\theta_{\varepsilon}-\theta_{\min }\right)^{-} d \sigma d t+\int_{0}^{t} \int_{\Gamma_{u}} u\left(\theta_{\varepsilon}-\theta_{\min }\right)^{-} d \sigma d \tau .
\end{aligned}
$$

Further, using the hypotheses, (4.5), and the fact that $\left|K\left(\theta_{\varepsilon}\right)\right| \leq M\left|\theta_{\varepsilon}-\theta_{\min }\right|$, we have

$$
\begin{aligned}
\frac{1}{2} \int_{\Omega} & {\left[\left(\theta_{\varepsilon}(t)-\theta_{\min }\right)^{-}\right]^{2} d x+\rho_{\alpha} \int_{0}^{t}\left\|\left(\theta_{\varepsilon}(t)-\theta_{\min }\right)^{-}\right\|_{V}^{2} d \tau } \\
\leq & \frac{M^{2}}{\rho_{\alpha}} \int_{0}^{t}\left\|\left(\theta_{\varepsilon}(\tau)-\theta_{\min }\right)^{-}\right\|^{2} d \tau+\frac{\rho_{\alpha}}{2} \int_{0}^{t}\left\|\left(\theta_{\varepsilon}(\tau)-\theta_{\min }\right)^{-}\right\|_{V}^{2} d \tau .
\end{aligned}
$$

This implies

$$
\left\|\left(\theta_{\varepsilon}(t)-\theta_{\min }\right)^{-}\right\|^{2} \leq \frac{2 M^{2}}{\rho_{\alpha}} \int_{0}^{t}\left\|\left(\theta_{\varepsilon}(\tau)-\theta_{\min }\right)^{-}\right\|^{2} d \tau,
$$

wherefrom we deduce according to Gronwall's lemma that $\left\|\left(\theta_{\varepsilon}(t)-\theta_{\min }\right)^{-}\right\|^{2}=0$, meaning that $\theta_{\varepsilon}(x, t) \geq \theta_{\min }$ a.e. on $\Omega$, for each $t \in[0, T]$.

Passing to the limit as $\varepsilon \rightarrow 0$, we obtain $\theta(x, t) \geq \theta_{\min }$ a.e. on $Q$.

Remark 4.7. In a similar way, it follows under certain conditions that $\theta(x, t) \leq \theta_{M}(t)=$ $P t+P_{0}$ a.e. on $\Omega$, for each $t$, where $\theta_{M}(t)$ is a solution to (4.26)-(4.27), $P \geq 0$, and $P_{0}=$ $\sup _{x \in \Omega} \theta_{0}(x)$. This result is implied by the proof of $\left\|\left(\theta_{\varepsilon}(t)-\theta_{M}(t)\right)^{+}\right\|^{2}=0$. 
Corollary 4.8. Assume $f \in L^{2}\left(0, T ; V^{\prime}\right), u \in L^{2}\left(0, T ; L^{2}\left(\Gamma_{u}\right)\right), f_{0} \in L^{2}\left(0, T ; L^{2}\left(\Gamma_{\alpha}\right)\right)$, and $\theta_{0} \in M_{\theta_{s}}$. Then problem (2.7) has a weak solution $h \in L^{2}(0, T ; V)$.

Proof. Under assumption (4.39), we obtain a solution $\theta$ satisfying the conclusions of Theorem 4.4, and we define

$$
h(x, t)=\left(C^{*}\right)^{-1}(\theta(x, t)), \quad \theta<\theta_{s}, h(x, t) \in[0,+\infty)=\left(C^{*}\right)^{-1}\left(\theta_{s}\right) .
$$

We will show that it is a weak solution to (2.7). We apply $K^{*}$ to $(4.73)$ and obtain

$$
K^{*}(h)=\beta^{*}(\theta), \quad \text { for } h<0, \theta<\theta_{s}, K^{*}(h) \in \beta^{*}\left(\theta_{s}\right), h \geq 0 .
$$

From (4.73) we get $\theta=C^{*}(h)$ and we introduce it in (4.23). We have

$$
\begin{aligned}
& \int_{Q}(\left.\frac{\partial C^{*}(h)}{\partial t} \phi+\nabla \eta \cdot \nabla \phi-K\left(C^{*}(h)\right) \frac{\partial \phi}{\partial x_{3}}\right) d x d t \\
&=\int_{Q} f \phi d x d t-\int_{\Sigma_{\alpha}}\left(\alpha \eta+f_{0}\right) \phi d \sigma d t-\int_{\Sigma_{u}} u \phi d \sigma d t
\end{aligned}
$$

for $\eta \in V$ such that $\eta \in \beta^{*}(\theta)$. After integrating the first term on the left-hand side with respect to $t$, we deduce that

$$
\begin{aligned}
\int_{Q}( & \left.-C^{*}(h) \phi_{t} d x d t+\nabla K^{*}(h) \cdot \nabla \phi-k(h) \frac{\partial \phi}{\partial x_{3}}\right) d x d t \\
& =\int_{\Omega} \phi(x, 0) C^{*}\left(h_{0}\right) d x-\int_{\Sigma_{\alpha}}\left(\alpha \eta+f_{0}\right) \phi d \sigma d t-\int_{\Sigma_{\alpha}} u \phi d \sigma d t-\int_{Q} f \phi d x d t .
\end{aligned}
$$

Since $\beta^{*}(\theta)=K^{*}\left(\left(C^{*}\right)^{-1}(\theta)\right) \in L^{2}(0, T ; V)$ and $K^{*}\left(\left(C^{*}\right)^{-1}(\theta)\right)$ satisfies

$$
\left(K^{*}\left(\left(C^{*}\right)^{-1}(\theta)\right)-K^{*}\left(\left(C^{*}\right)^{-1}(\bar{\theta})\right)\right)(\theta-\bar{\theta}) \geq K_{r}\left(\left(C^{*}\right)^{-1}(\theta)-\left(C^{*}\right)^{-1}(\bar{\theta})\right)^{2},
$$

for all $\theta, \bar{\theta} \leq \theta_{s}$, it follows that $h=\left(C^{*}\right)^{-1}(\theta) \in L^{2}(0, T ; V)$.

\section{Existence of free boundary}

To prove that there exists a free boundary $s=s\left(t, x_{1}, x_{2}\right)$ that determines a strict delimitation of the domains $Q_{+}$and $Q_{-}$with $Q_{+}$above $Q_{-}$, the idea is to prove that the function $h$ is monotonically decreasing with respect to $x_{3}$, that is, $w_{h}=\partial h / \partial x_{3} \leq 0$. Consequently, the equation $h(x, t)=0$ can be solved with respect to $x_{3}$ and has a unique solution $x_{3}=s\left(t, x_{1}, x_{2}\right)$.

To come to this end, we first need to have some supplementary regularities for the solution $\theta_{\varepsilon}$ to the approximating problem (4.26)-(4.27), considered for a smoother approximation of class $C^{3}(\mathbb{R})$. 
Proposition 5.1. Let

$$
\begin{gathered}
f \in W^{1,2}\left(0, T ; L^{2}(\Omega)\right) \cap L^{\infty}(Q), \quad u \in W^{1,2}\left(0, T ; L^{2}\left(\Gamma_{u}\right)\right) \cap L^{\infty}\left(\Sigma_{u}\right), \\
f_{0} \in W^{1,2}\left(0, T ; L^{2}\left(\Gamma_{\alpha}\right)\right) \cap L^{\infty}\left(\Sigma_{\alpha}\right), \\
u \leq 0 \quad \text { on } \Sigma_{u}, \\
\theta_{0} \in L^{2}(\Omega) \quad \text { such that } \beta_{\varepsilon}^{*}\left(\theta_{0}\right) \in H^{1}(\Omega) .
\end{gathered}
$$

Then, the solution to the approximating problem (4.26)-(4.27) has the supplementary property

$$
\beta_{\varepsilon}^{*}\left(\theta_{\varepsilon}\right) \in L^{2}\left(0, T ; H^{2}(\Omega)\right) .
$$

Proof. Under the hypotheses (5.1) and (5.3) which are stronger than those imposed in Proposition 4.2 , it follows that $\theta_{\varepsilon}$ is a unique strong solution to the approximating problem (4.26)-(4.27) and satisfies the conclusions of Proposition 4.2. Moreover, the requirement (5.3) implies that $\theta_{\varepsilon} \in D\left(A_{\varepsilon}\right)$.

Consider again the Cauchy problem (4.26)-(4.27). By a similar argument as done for the exact problem, its strong solution $\theta_{\varepsilon}$ is a solution to the boundary value problem

$$
\begin{gathered}
\frac{\partial \theta_{\varepsilon}}{\partial t}-\Delta \beta_{\varepsilon}^{*}\left(\theta_{\varepsilon}\right)+\frac{\partial K\left(\theta_{\varepsilon}\right)}{\partial x_{3}}=f \quad \text { in } Q, \\
\theta_{\varepsilon}(x, 0)=\theta_{0}(x) \quad \text { in } \Omega, \\
\left(K\left(\theta_{\varepsilon}\right) i_{3}-\nabla \beta_{\varepsilon}^{*}\left(\theta_{\varepsilon}\right)\right) \cdot v=u \text { on } \Sigma_{u}, \\
\left(K\left(\theta_{\varepsilon}\right) i_{3}-\nabla \beta_{\varepsilon}^{*}\left(\theta_{\varepsilon}\right)\right) \cdot v=\alpha \beta_{\varepsilon}^{*}\left(\theta_{\varepsilon}\right)+f_{0} \quad \text { on } \Sigma_{\alpha} .
\end{gathered}
$$

Since the function $\beta_{\varepsilon}^{*}$ is continuous and monotonically increasing, we may define its inverse. Moreover, we assumed that $\beta_{\varepsilon}^{*}$ is in this case of class $C^{3}(\mathbb{R})$, so that its first derivative $\beta_{\varepsilon}$ is bounded on $\mathbb{R}$ and satisfies

$$
\rho \leq \beta_{\varepsilon}\left(\theta_{\varepsilon}\right) \leq \rho_{\varepsilon}<\infty
$$

for each $\varepsilon>0$. Hence, denoting $\eta=\beta_{\varepsilon}^{*}\left(\theta_{\varepsilon}\right)$ with $\eta(0)=\beta_{\varepsilon}^{*}\left(\theta_{0}\right)$, we have

$$
\begin{gathered}
\theta_{\varepsilon}=\left(\beta_{\varepsilon}^{*}\right)^{-1}(\eta), \\
\frac{\partial \theta_{\varepsilon}}{\partial t}=\frac{\partial}{\partial t}\left(\left(\beta_{\varepsilon}^{*}\right)^{-1}(\eta)\right)=\omega(t) \frac{\partial \eta}{\partial t},
\end{gathered}
$$

and $\zeta(\eta)=K\left(\left(\beta_{\varepsilon}^{*}\right)^{-1}(\eta)\right)$, where $\omega(\eta)=1 / \beta_{\varepsilon}\left(\left(\beta_{\varepsilon}^{*}\right)^{-1}(\eta)\right)$. We also know that

$$
0<\rho_{\varepsilon}^{1} \leq \omega(\eta) \leq \rho_{2}<\infty, \quad \rho_{\varepsilon}^{1}=\frac{1}{\rho_{\varepsilon}}, \rho_{2}=\frac{1}{\rho} .
$$

Using the conclusions of Proposition 4.2, we deduce that

$$
\eta \in L^{\infty}(0, T, V), \quad \omega(\eta) \eta_{t} \in L^{\infty}\left(0, T, V^{\prime}\right), \quad \zeta(\eta) \in L^{\infty}(0, T, V) .
$$


Therefore, because $\omega(\eta)$ is bounded, we still obtain $\eta_{t} \in L^{\infty}\left(0, T ; V^{\prime}\right)$. We introduce (5.7) in (5.5) and obtain

$$
\begin{gathered}
\omega(\eta) \frac{\partial \eta}{\partial t}-\Delta \eta+\frac{\partial \zeta(\eta)}{\partial x_{3}}=f \quad \text { in } Q, \\
\eta_{0}(x)=\eta(x, 0)=\beta_{\varepsilon}^{*}\left(\theta_{0}\right) \quad \text { in } \Omega, \\
\left(\zeta(\eta) i_{3}-\nabla \eta\right) \cdot v=u \quad \text { on } \Sigma_{u}, \quad\left(\zeta(\eta) i_{3}-\nabla \eta\right) \cdot v=\alpha \eta+f_{0} \quad \text { on } \Sigma_{\alpha} .
\end{gathered}
$$

We multiply equation (5.11) by $\eta_{t}\left(\eta_{t}=\partial \eta / \partial t\right)$ and integrate over $\Omega \times(0, t)$. We obtain

$$
\begin{gathered}
\int_{0}^{t} \int_{\Omega} \omega(\eta) \eta_{\tau}^{2} d \tau d x+\frac{1}{2} \int_{0}^{t} \frac{d}{d t}\left(\int_{\Omega}|\nabla \eta(\tau)|^{2} d x\right) d \tau-\int_{0}^{t} \int_{\Omega} \zeta(\eta) \frac{\partial \eta_{\tau}}{\partial x_{3}} d \tau d x \\
=-\int_{0}^{t} \int_{\Gamma_{u}} u \eta_{\tau} d \sigma d \tau-\int_{0}^{t}\left(\alpha \eta+f_{0}\right) \eta_{\tau} d \sigma d \tau+\int_{0}^{t} \int_{\Omega} f \eta_{\tau} d x d \tau .
\end{gathered}
$$

We calculate

$$
\begin{aligned}
-\int_{0}^{t} \int_{\Gamma_{u}} u \eta_{\tau} d \sigma d \tau & =-\int_{0}^{t} \int_{\Gamma_{u}}\left(\frac{\partial}{\partial t}(u \eta)-\eta \frac{\partial u}{\partial \tau}\right) d \sigma d \tau \\
& =-\int_{\Gamma_{u}} u(t) \eta(t) d \sigma+\int_{\Gamma_{u}} u_{0} \eta_{0} d \sigma+\int_{0}^{t} \int_{\Gamma_{u}} \eta \frac{\partial u}{\partial \tau} d \sigma d \tau
\end{aligned}
$$

Proceeding in the same manner for all other terms on the right-hand side, we get after some calculations roughly the estimate

$$
\begin{aligned}
& \int_{0}^{t} \int_{\Omega} \eta_{\tau}^{2} d x d \tau+\int_{\Omega}|\nabla \eta(\tau)|^{2} d x \\
& \leq c_{0} \int_{\Omega}\left|\nabla \eta_{0}\right|^{2} d x+\|\zeta(\eta(t))\|\left\|\frac{\partial \eta(t)}{\partial x_{3}}\right\|+\left\|\zeta\left(\eta_{0}\right)\right\|\left\|\frac{\partial \eta_{0}}{\partial x_{3}}\right\| \\
& \quad+\int_{0}^{t}\left\|\frac{\partial \zeta(\eta(\tau))}{\partial x_{3}}\right\|\left\|\frac{\partial \eta(\tau)}{\partial x_{3}}\right\| d \tau+\int_{0}^{t}\|\eta(\tau)\|_{L^{2}\left(\Gamma_{u}\right)}\left\|\frac{\partial u(\tau)}{\partial \tau}\right\|_{L^{2}\left(\Gamma_{u}\right)} d \tau \\
&+\int_{0}^{t}\|\eta(\tau)\|_{L^{2}\left(\Gamma_{\alpha}\right)}\left\|\frac{d f_{0}(\tau)}{d \tau}\right\|\left\|_{L^{2}\left(\Gamma_{\alpha}\right)} d \tau+\right\| u(t)\left\|_{L^{2}\left(\Gamma_{u}\right)}\right\| \eta(t) \|_{L^{2}\left(\Gamma_{u}\right)} \\
&+\left\|u_{0}\right\|\left\|_{L^{2}\left(\Gamma_{u}\right)}\right\| \eta_{0}\left\|_{L^{2}\left(\Gamma_{u}\right)}+\right\| f_{0}(t)\left\|_{L^{2}\left(\Gamma_{\alpha}\right)}\right\| \eta(t)\|\|_{L^{2}\left(\Gamma_{\alpha}\right)}+\left\|f_{0}(0)\right\|_{L^{2}\left(\Gamma_{\alpha}\right)}\left\|\eta_{0}\right\|_{L^{2}\left(\Gamma_{\alpha}\right)} \\
&+\|f(t)\|\|\eta(t)\|+\|f(0)\|\left\|\eta_{0}\right\|+\int_{0}^{t}\|\eta(\tau)\|\left\|\frac{\partial f(\tau)}{\partial \tau}\right\| d \tau .
\end{aligned}
$$

But, from the hypotheses, it follows that $u_{0}, f_{0}(0), f(0)$, and $\eta_{0}$ make sense and finally we conclude that the right-hand side in (5.16) is bounded. This implies that

$$
\eta_{t} \in L^{2}\left(0, T ; L^{2}(\Omega)\right), \quad \eta \in L^{\infty}\left(0, T ; H^{1}(\Omega)\right) .
$$


A little problem in the previous calculation is that we do not know a priori that $\eta_{t} \in L^{2}\left(0, T ; L^{2}(\Omega)\right)$. Hence, rigorously, $(5.11)$ should have been approximated by a finitedifference equation for $(\eta(t+\delta)-\eta(t)) / \delta$, and in the same manner, the result obtained would have been

$$
\int_{0}^{t}\|\eta(\tau+\delta)-\eta(\tau)\|^{2} d \tau \leq c \delta^{2}
$$

that implies $\eta \in W^{1,2}\left(0, T ; L^{2}(\Omega)\right)$ (see [3, page 21]).

Then, from (5.11), we have that

$$
\|\Delta \eta\| \leq\left\|\omega(\eta) \frac{\partial \eta}{\partial t}\right\|+\left\|\frac{\partial \zeta(\eta)}{\partial x_{3}}\right\|+\|f\|
$$

and we can deduce that

$$
\Delta \eta \in L^{2}\left(0, T ; L^{2}(\Omega)\right)
$$

and consequently (taking into account the boundary conditions)

$$
\eta \in L^{2}\left(0, T ; H^{2}(\Omega)\right) .
$$

Finally we have to keep in mind that $\theta_{\varepsilon} \in L^{\infty}\left(0, T ; H^{1}(\Omega)\right)$ and $\beta_{\varepsilon}^{*}\left(\theta_{\varepsilon}\right) \in L^{2}(0, T$; $\left.H^{2}(\Omega)\right) \cap L^{\infty}\left(0, T ; H^{1}(\Omega)\right)$.

Proposition 5.2. Under the hypotheses of Proposition 5.1,

$$
\theta_{\varepsilon} \in L^{4 / 3}\left(0, T ; H^{2}(\Omega)\right) .
$$

Proof. We start from (5.7) and calculate the partial derivative of $\theta_{\varepsilon}$, denoted further $\theta_{x_{i}}$ (we omit the subscript $\varepsilon$ ) with respect to $x_{i}$. First we remind the reader that we work with the smooth approximate of class $C^{3}$, whose derivatives up to the third order (denoted $\beta_{\varepsilon}$, $\beta_{\varepsilon}^{\prime}$, and $\left.\beta_{\varepsilon}^{\prime \prime}\right)$ are bounded. We have

$$
\theta_{x_{i}}=\frac{\eta_{x_{i}}}{\beta_{\varepsilon}\left(\left(\beta_{\varepsilon}^{*}\right)^{-1}(\eta)\right)} \in L^{2}\left(0, T ; H^{1}(\Omega)\right) \cap L^{\infty}\left(0, T ; L^{2}(\Omega)\right) .
$$

Then

$$
\theta_{x_{i} x_{j}}=\frac{\eta_{x_{i} x_{j}} \beta_{\varepsilon}\left(\left(\beta_{\varepsilon}^{*}\right)^{-1}(\eta)\right)-\eta_{x_{i}} \eta_{x_{j}} \beta_{\varepsilon}^{\prime}\left(\left(\beta_{\varepsilon}^{*}\right)^{-1}(\eta)\right) / \beta_{\varepsilon}\left(\left(\beta_{\varepsilon}^{*}\right)^{-1}(\eta)\right)}{\left[\beta_{\varepsilon}\left(\left(\beta_{\varepsilon}^{*}\right)^{-1}(\eta)\right)\right]^{2}}
$$

and we need to estimate its norm. Since $\eta_{x_{i} x_{j}} \in L^{2}\left(0, T ; L^{2}(\Omega)\right)$, we will deal only with the product $\eta_{x_{i}} \eta_{x_{j}}$. From the previous proposition we have $\eta \in L^{2}\left(0, T ; H^{2}(\Omega)\right)$, which implies the following sequence:

$$
\eta_{x_{i}}(t) \in H^{1}(\Omega) \subset L^{6}(\Omega) \subset L^{4}(\Omega) \subset L^{2}(\Omega), \quad \forall N \leq 3 .
$$


Now $\eta_{x_{i}}(t) \in L^{4}(\Omega)$ implies $\eta_{x_{i}}^{2}(t) \in L^{2}(\Omega)$ so that we can write

$$
\left\|\eta_{x_{i}}(t) \eta_{x_{j}}(t)\right\|^{2}=\int_{\Omega} \eta_{x_{i}}^{2}(t) \eta_{x_{j}}^{2}(t) d x \leq\left(\int_{\Omega} \eta_{x_{i}}^{4}(t) d x\right)^{1 / 2}\left(\int_{\Omega} \eta_{x_{j}}^{4}(t) d x\right)^{1 / 2} .
$$

But $\eta_{x_{i}}(t) \in L^{6}(\Omega)$ implies $\eta_{x_{i}}^{3}(t) \in L^{2}(\Omega)$ so that we have

$$
\int_{\Omega} \eta_{x_{i}}^{4}(t) d x=\int_{\Omega} \eta_{x_{i}}(t) \eta_{x_{i}}^{3}(t) d x \leq\left\|\eta_{x_{i}}(t)\right\|\left\|\eta_{x_{i}}^{3}(t)\right\|
$$

or

$$
\int_{\Omega} \eta_{x_{i}}^{4}(t) d x \leq\|\eta(t)\|_{H^{1}(\Omega)}\|\eta(t)\|_{H^{2}(\Omega)}^{3}
$$

Then we obtain

$$
\begin{aligned}
\left\|\theta_{x_{i} x_{j}}(t)\right\|^{2} & \leq d_{0}\left\|\eta_{x_{i} x_{j}}(t)\right\|^{2}+d_{1}\left\|\eta_{x_{i}}(t) \eta_{x_{j}}(t)\right\|^{2} \\
& \leq d_{0}\|\eta(t)\|_{H^{2}(\Omega)}^{2}+d_{1}\|\eta(t)\|_{H^{1}(\Omega)}\|\eta(t)\|_{H^{2}(\Omega)}^{3},
\end{aligned}
$$

and therefore we get

$$
\left\|\theta_{\varepsilon}(t)\right\|_{H^{2}(\Omega)}^{2} \leq d_{2}\|\eta(t)\|_{H^{2}(\Omega)}+d_{3}\|\eta(t)\|_{H^{2}(\Omega)}^{3} .
$$

Finally, this yields

$$
\int_{0}^{t}\left\|\theta_{\varepsilon}(\tau)\right\|_{H^{2}(\Omega)}^{4 / 3} d \tau \leq d_{4} \int_{0}^{t}\|\eta(\tau)\|_{H^{2}(\Omega)}^{2} d \tau<\infty
$$

with $d_{0}, d_{1}, d_{2}, d_{3}$, and $d_{4}$ some constants.

We now pass to the proof of the monotonicity of the partial derivative of $\theta$ with respect to $x_{3}$.

A better insight can be gained in one dimension, so that we will prove the result for the case $N=1$. In this case we denote $z=x_{3}$, the domain $\Omega$ becomes $\Omega=(0, L), \Gamma_{u}=\{z ; z=$ $0\}, \Gamma_{\alpha}=\{z ; z=L\}, \alpha(L)=\alpha=\alpha_{M}, f_{0}(x, t)=f_{0}(t)$, and system (3.4) reads

$$
\begin{gathered}
C(h) h_{t}-\left(k(h) h_{z}\right)_{z}+(k(h))_{z}=f \quad \text { in } Q_{-}=\{z ; s(t)<z<L\}, \\
-K_{s} \Delta h=f \quad \text { in } Q_{+}=\{z ; 0<z<s(t)\}, \\
h(z, 0)=h_{0}(z) \quad \text { in }(0, L), \\
q^{+}(s(t), t)=q^{-}(s(t), t), \quad h^{+}(s(t), t)=h^{-}(s(t), t), \\
K_{s}-K_{s} h_{z}(0, t)=-u(t) \stackrel{\text { notation }}{=} u_{-}(t), \\
k(h(L, t))-k(h(L, t)) h_{z}(L, t)=\alpha K^{*}(h(L, t))+f_{0}(t) .
\end{gathered}
$$

Solving this problem, one determines the free boundary $z=s(t)$ from the equation $h(z, t)=0$. The subscript $z$ means the partial derivative with respect to $z$.

Obviously, in order to ensure the existence of a free boundary that determines a clear separation of the saturated region from the unsaturated one, some conditions have to be fulfilled and this is presented in the following result. 
Proposition 5.3. Let $N=1, K_{s} \leq \alpha_{M} K_{s}^{*}$,

$$
\begin{gathered}
f \in W^{1,2}\left(0, T ; L^{2}(\Omega)\right) \cap L^{\infty}(Q), \quad u \in W^{1,2}\left(0, T ; L^{2}\left(\Gamma_{u}\right)\right) \cap L^{\infty}\left(\Sigma_{u}\right), \\
f_{0} \in W^{1,2}\left(0, T ; L^{2}\left(\Gamma_{\alpha}\right)\right) \cap L^{\infty}\left(\Sigma_{\infty}\right), \quad \theta_{0} \in L^{2}(\Omega), \quad \text { such that } \beta_{\varepsilon}^{*}\left(\theta_{0}\right) \in H^{1}(\Omega), \\
\frac{\partial \theta_{0}}{\partial z}(z, 0) \leq 0 \quad \text { in } \Omega, \quad f_{z}(z, t) \leq 0 \quad \text { in } Q, \quad u_{-}(t) \geq K_{s}, \\
f_{0}(t) \geq \sup _{r \in\left[0, \theta_{s}\right]}\left\{K(r)-\alpha_{M} \stackrel{\circ}{*}(r)\right\} .^{*}
\end{gathered}
$$

Then $w=\partial \theta / \partial z$ is negative a.e. on $(0, L) \times(0, T)$ and $z \rightarrow \theta(z, t)$ is monotonically decreasing on $[0, T]$, for each $t \in[0, T]$.

Proof. We mean by $\beta^{*}$ the minimal section of $\beta^{*}$. By Proposition 5.1, without any loss of generality, we may assume that $\theta_{\varepsilon}$ is smooth enough and for that we work with the approximate which has the property that $\beta_{\varepsilon}^{\prime \prime} \in C^{0}(\mathbb{R})$.

By the hypotheses, it follows that $\theta_{\varepsilon}$ is a solution satisfying the conclusions of Propositions 5.1 and 5.2.

We denote $w_{\varepsilon}=\partial \theta_{\varepsilon} / \partial z$ and note that by assumption $w_{\varepsilon}(z, 0) \leq 0$. From the boundary conditions we have

$$
\begin{gathered}
K\left(\theta_{\varepsilon}\right)-\beta_{\varepsilon}\left(\theta_{\varepsilon}\right) w_{\varepsilon}=u_{-} \text {for } z=0, \\
K\left(\theta_{\varepsilon}\right)-\beta_{\varepsilon}\left(\theta_{\varepsilon}\right) w_{\varepsilon}=\alpha \beta_{\varepsilon}^{*}\left(\theta_{\varepsilon}\right)+f_{0} \quad \text { for } z=L .
\end{gathered}
$$

If we assume that saturation begins from above, then we will necessarily have that $u_{-}(t) \geq$ $K_{s} \geq K\left(\theta_{\varepsilon}(0, t)\right)$. Then we have

$$
w_{\varepsilon}(0, t)=\frac{K\left(\theta_{\varepsilon}(0, t)\right)-u_{-}(t)}{\beta_{\varepsilon}\left(\theta_{\varepsilon}(0, t)\right)} \leq 0
$$

implying that $w_{\varepsilon}^{+}(0, t)=0$.

Then, if we assume that for $z=L$ we have

$$
f_{0}(t) \geq \sup _{r \in\left[0, \theta_{s}\right]}\left(K(r)-\alpha_{M} \stackrel{\circ}{*}^{*}(r)\right) \geq K(r)-\alpha_{M} \beta^{*}(r), \quad \forall r \in\left[0, \theta_{s}\right],
$$

we will have from the boundary condition on $z=L$ and taking into account that $K_{s} \leq$ $\alpha_{M} K_{s}^{*}$ that

$$
w_{\varepsilon}(L, t)=\frac{K\left(\theta_{\varepsilon}(L, t)\right)-\left(\alpha \beta_{\varepsilon}^{*}(\theta(L, t))+f_{0}(t)\right)}{\beta_{\varepsilon}\left(\theta_{\varepsilon}(L, t)\right)} \leq 0,
$$

that is, $w_{\varepsilon}^{+}(L, t)=0$.

We differentiate (5.5) with respect to $z$ :

$$
\frac{\partial w_{\varepsilon}}{\partial t}-\Delta\left(\beta_{\varepsilon}\left(\theta_{\varepsilon}\right) w_{\varepsilon}\right)+\frac{\partial}{\partial z}\left(K^{\prime}\left(\theta_{\varepsilon}\right) w_{\varepsilon}\right)=f_{z} .
$$




\section{A free boundary problem}

Then we multiply it by $w_{\varepsilon}^{+}$and integrate over $\Omega \times(0, t)$. We have

$$
\begin{aligned}
& \frac{1}{2}\left\|w_{\varepsilon}^{+}(t)\right\|^{2}+\int_{0}^{t} \int_{0}^{L}\left[\frac{\partial}{\partial z}\left(\beta_{\varepsilon}\left(\theta_{\varepsilon}\right) w_{\varepsilon}\right) \frac{\partial w_{\varepsilon}^{+}}{\partial z}-K^{\prime}\left(\theta_{\varepsilon}\right) w_{\varepsilon} \frac{\partial w_{\varepsilon}^{+}}{\partial z}\right] d z d \tau \\
& +\left.\int_{0}^{t}\left[K^{\prime}\left(\theta_{\varepsilon}\right) w_{\varepsilon}-\frac{\partial}{\partial z}\left(\beta_{\varepsilon}\left(\theta_{\varepsilon}\right) w_{\varepsilon}\right)\right] w_{\varepsilon}^{+}\right|_{z=0} ^{z=L} d \tau=\int_{0}^{t} \int_{0}^{L} f_{z} w_{\varepsilon}^{+} d z d \tau .
\end{aligned}
$$

But, using Stampacchia's lemma and $w^{2}=\left(w^{+}\right)^{2}$, we have

$$
\begin{aligned}
\int_{0}^{t} \int_{0}^{L} \frac{\partial}{\partial z}\left(\beta_{\varepsilon}\left(\theta_{\varepsilon}\right) w_{\varepsilon}\right) \frac{\partial w_{\varepsilon}^{+}}{\partial z} d z d \tau & =\int_{0}^{t} \int_{0}^{L}\left(\beta_{\varepsilon}\left(\theta_{\varepsilon}\right) w_{\varepsilon}^{2}+\beta_{\varepsilon}\left(\theta_{\varepsilon}\right) \frac{\partial w_{\varepsilon}}{\partial z}\right) \frac{\partial w_{\varepsilon}^{+}}{\partial z} d z d \tau \\
& =\int_{0}^{t} \int_{0}^{L} \frac{1}{3}\left(\beta_{\varepsilon}^{\prime}\left(\theta_{\varepsilon}\right) \frac{\partial}{\partial z}\left(w_{\varepsilon}^{+}\right)^{3}+\beta_{\varepsilon}\left(\theta_{\varepsilon}\right)\left(\frac{\partial w_{\varepsilon}^{+}}{\partial z}\right)^{2}\right] d z d \tau
\end{aligned}
$$

\section{Moreover}

$$
\int_{0}^{t} \int_{0}^{L} \beta_{\varepsilon}^{\prime}\left(\theta_{\varepsilon}\right) \frac{\partial}{\partial z}\left(w_{\varepsilon}^{+}\right)^{3} d z d \tau=\left.\int_{0}^{t} \beta_{\varepsilon}^{\prime}\left(\theta_{\varepsilon}\right)\left(w_{\varepsilon}^{+}\right)^{3}\right|_{0} ^{L} d \tau-\int_{0}^{t} \int_{0}^{L} \beta_{\varepsilon}^{\prime \prime}\left(\theta_{\varepsilon}\right)\left(w_{\varepsilon}^{+}\right)^{4} d z d \tau
$$

and $K^{\prime}\left(\theta_{\varepsilon}\right) \leq M$, so we get

$$
\begin{aligned}
\frac{1}{2}\left\|w_{\varepsilon}^{+}(t)\right\|^{2}+\rho \int_{0}^{t} \int_{0}^{L}\left(\frac{\partial w_{\varepsilon}^{+}}{\partial z}\right)^{2} d z d \tau \leq & \frac{1}{2} \int_{0}^{t}\left(\rho\left\|\frac{\partial w_{\varepsilon}^{+}}{\partial z}\right\|^{2}+\frac{M^{2}}{\rho}\left\|w_{\varepsilon}^{+}(t)\right\|^{2}\right) d \tau \\
& +\frac{1}{3} \int_{0}^{t} \int_{0}^{L} \beta_{\varepsilon}^{\prime \prime}\left(\theta_{\varepsilon}\right)\left(w_{\varepsilon}^{+}\right)^{4} d z d \tau .
\end{aligned}
$$

Finally, we obtain

$$
\begin{aligned}
& \frac{1}{2}\left\|w_{\varepsilon}^{+}(t)\right\|^{2}+\frac{\rho}{2} \int_{0}^{t} \int_{0}^{L}\left(\frac{\partial w_{\varepsilon}^{+}}{\partial z}\right)^{2} d z d \tau \\
& \quad \leq \frac{M^{2}}{2 \rho} \int_{0}^{t}\left\|w_{\varepsilon}^{+}(\tau)\right\|^{2} d \tau+\frac{1}{3} \int_{0}^{t} \int_{0}^{L} \beta_{\varepsilon}^{\prime \prime}\left(\theta_{\varepsilon}\right)\left(w_{\varepsilon}^{+}(\tau)\right)^{4} d \tau
\end{aligned}
$$

where $\beta_{\varepsilon}^{\prime \prime}\left(\theta_{\varepsilon}\right)$ is bounded by a constant denoted $\beta_{M}^{\prime \prime}$. We now write the following relation:

$$
\frac{1}{2}\left(w_{\varepsilon}^{+}(t)\right)^{2}=\int_{0}^{z} w_{\varepsilon}^{+}(t) \frac{\partial w_{\varepsilon}^{+}(t)}{\partial s} d s \leq\left(\int_{0}^{L}\left(w_{\varepsilon}^{+}(t)\right)^{2} d z\right)^{1 / 2}\left(\int_{0}^{L}\left(\frac{\partial w_{\varepsilon}^{+}(t)}{\partial z}\right)^{2} d z\right)^{1 / 2} .
$$


By Proposition 5.1, we have that $\nabla \theta_{\varepsilon} \in L^{\infty}\left(0, T ; H^{1}(\Omega)\right)$, implying that the same result is inherited by $\theta_{\varepsilon}^{+}$. Then $w_{\varepsilon}^{+} \in L^{\infty}\left(0, T ; H^{1}(\Omega)\right)$. We denote

$$
\gamma(t)=\left(\int_{0}^{L}\left(\frac{\partial w_{\varepsilon}^{+}(t)}{\partial z}\right)^{2} d z\right)^{1 / 2}=\left\|\frac{\partial w_{\varepsilon}^{+}(t)}{\partial z}\right\|=\left\|\frac{\partial^{2} \theta_{\varepsilon}^{+}(t)}{\partial z^{2}}\right\| \leq\left\|\theta_{\varepsilon}(t)\right\|_{H^{2}(\Omega)},
$$

so

$$
\frac{1}{2}\left(w_{\varepsilon}^{+}(t)\right)^{2} \leq\left\|w_{\varepsilon}^{+}(t)\right\| \gamma(t) \leq c_{3} \gamma(t)
$$

In (5.43) we still can write the last term on the right-hand side as

$$
\begin{aligned}
\int_{0}^{t} \int_{0}^{L} \beta_{\varepsilon}^{\prime \prime}\left(\theta_{\varepsilon}\right)\left(w_{\varepsilon}^{+}(\tau)\right)^{4} d z d \tau & \leq \beta_{M}^{\prime \prime} \int_{0}^{t} \int_{0}^{L}\left(w_{\varepsilon}^{+}(\tau)\right)^{2}\left(w_{\varepsilon}^{+}(\tau)\right)^{2} d z d \tau \\
& \leq c_{4} \int_{0}^{t} \int_{\Omega} \gamma(\tau)\left(w_{\varepsilon}^{+}(\tau)\right)^{2} d z d \tau,
\end{aligned}
$$

where, by Proposition 5.2, it follows that $\gamma \in L^{4 / 3}(0, T) \subset L^{1}(0, T)$.

Recalling again (5.43), we finally get

$$
\frac{1}{2}\left\|w_{\varepsilon}^{+}(t)\right\|^{2}+\frac{\rho}{2} \int_{0}^{t} \int_{0}^{L}\left(\frac{\partial w_{\varepsilon}^{+}}{\partial z}\right)^{2} d z d \tau \leq \int_{0}^{t}\left(\frac{M^{2}}{2 \rho}+c_{4} \gamma(\tau)\right)\left\|w_{\varepsilon}^{+}(\tau)\right\|^{2} d \tau .
$$

Further, we apply Gronwall's lemma and we obtain

$$
w_{\varepsilon}(x, t) \leq 0, \quad \forall t \in(0, T) .
$$

Since the solution $\theta_{\varepsilon}$ having all these properties tends strongly to $\theta$ in $L^{2}(Q)$, by passing to the limit as $\varepsilon \rightarrow 0$, we obtain that $w(x, t) \leq 0$.

The conclusion is that if we define for each $t \in[0, T]$,

$$
s(t)=\sup \left\{z ; \theta(z, t)<\theta_{s}\right\}
$$

the curve $z=s(t)$ separates the regions $Q_{-}$and $Q_{+}$.

\section{Uniqueness of the weak and smooth solution}

For weak solutions only, the uniqueness is not rather obvious. But if the weak solution is sufficiently smooth such that it may imply a smooth separation surface, the uniqueness can be proved. 
Under the conditions of Proposition 5.3, in the 1D case the sets $\{(z, t) ; h(z, t)<0\}$, $\{(z, t) ; h(z, t)>0\}$, and $\{(z, t) ; h(z, t)=0\}$ are open and it follows that any solution $h$ satisfies

$$
\begin{gathered}
h_{z z}=-\frac{f}{K_{s}}, \quad 0<z<s(t), \\
-h_{z}(0, t)=\frac{u_{-}(t)-K_{s}}{K_{s}}, \quad 0 \leq t \leq T, \\
h(s(t), t)=0 .
\end{gathered}
$$

Hence

$$
h_{z}(z, t)=\frac{K_{s}-u_{-}(t)}{K_{s}}-\frac{1}{K_{s}} \int_{0}^{z} f(\xi, t) d \xi
$$

and finally

$$
h(z, t)=\frac{u_{-}(t)-K_{s}}{K_{s}}(s(t)-z)+\frac{1}{K_{s}} \int_{z}^{s(t)} d \zeta \int_{0}^{\zeta} f(\xi, t) d \xi, \quad 0<z<s(t) .
$$

Since $s(t)$ is defined by $\theta(s(t), t)=\theta_{s}$ and $\theta$ is unique, it follows that $h$ is uniquely defined on $0<z<s(t)$, that is, in $\{(z, t) ; h(z, t)>0\}$.

In $Q_{-}$we have

$$
\left(C^{*}(h)\right)_{t}-\Delta K^{*}(h)+(K(h))_{z}=f \quad \text { in } Q_{-},
$$

with flux boundary conditions on $\{z ; z=L\} \cup\{z ; z=s(t)\}$. Equivalently

$$
\theta_{t}-\Delta \beta^{*}(h)+(K(h))_{z}=f \quad \text { in }\left\{\theta<\theta_{s}\right\}
$$

Since $C^{*}(h)$ is uniquely defined, so is $h$.

\section{References}

[1] H. W. Alt, S. Luckhaus, and A. Visintin, On nonstationary flow through porous media, Ann. Mat. Pura Appl. (4) 136 (1984), 303-316.

[2] C. Baiocchi, Su un problema di frontiera libera connesso a questioni di idraulica, Ann. Mat. Pura Appl. (4) 92 (1972), 107-127 (Italian).

[3] V. Barbu, Nonlinear semigroups and differential equations in Banach spaces, Editura AcademieiNoordhoff International Publishing, Bucharest-Leyden, 1976.

[4] , Partial Differential Equations and Boundary Value Problems, Mathematics and Its Applications, vol. 441, Kluwer Academic Publishers, Dordrecht, 1998.

[5] V. Barbu and G. Marinoschi, Controlling the volumetric water content jump in a stratified unsaturated soil, Nonlinear Analysis and Applications: To V. Lakshmikantham on His 80th Birthday. Vol. 1, 2 (A. Ravi and D. O’Regan, eds.), Kluwer Academic Publishers, Dordrecht, 2003, pp. 241-258.

[6] Existence for a time-dependent rainfall infiltration model with a blowing up diffusivity, Nonlinear Anal. Real World Appl. 5 (2004), no. 2, 231-245.

[7] J. Bear, Hydraulics of Groundwater, McGraw-Hill, New York, 1979. 
[8] I. Borsi, A. Farina, and A. Fasano, On the infiltration of rain water through the soil with runoff of the excess water, Nonlinear Anal. Real World Appl. 5 (2004), no. 5, 763-800.

[9] G. Gilardi, A new approach to evolution free boundary problems, Comm. Partial Differential Equations 4 (1979), no. 10, 1099-1122.

[10] J.-L. Lions, Quelques Méthodes de Résolution des Problèmes aux Limites Non Linéaires, Dunod, Paris, 1969.

[11] G. Marinoschi, Nonlinear infiltration with a singular diffusion coefficient, Differential Integral Equations 16 (2003), no. 9, 1093-1110.

[12] A. Torelli, Su un problema a frontiera libera di evoluzione, Boll. Un. Mat. Ital. (4) 11 (1975), no. 3, 559-570 (Italian).

[13] A. Visintin, Existence results for some free boundary filtration problems, Ann. Mat. Pura Appl. (4) 124 (1980), 293-320.

[14] I. White and P. Broadbridge, Constant rate rainfall infiltration: a versatile nonlinear model. 1. Analytic solution, Water Resour. Res. 24 (1988), no. 1, 145-154.

Gabriela Marinoschi: Institute of Mathematical Statistics and Applied Mathematics, Romanian Academy, P.O. Box 1-24, 050711 Bucharest, Romania

E-mail address: gmarino@acad.ro 


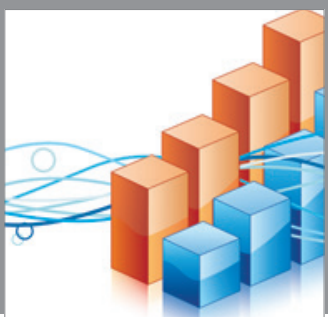

Advances in

Operations Research

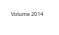

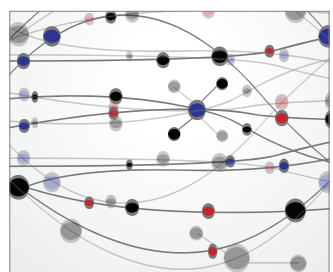

\section{The Scientific} World Journal
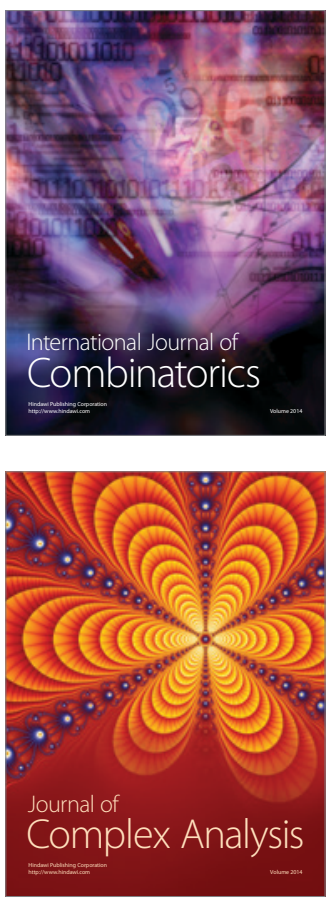

International Journal of

Mathematics and

Mathematical

Sciences
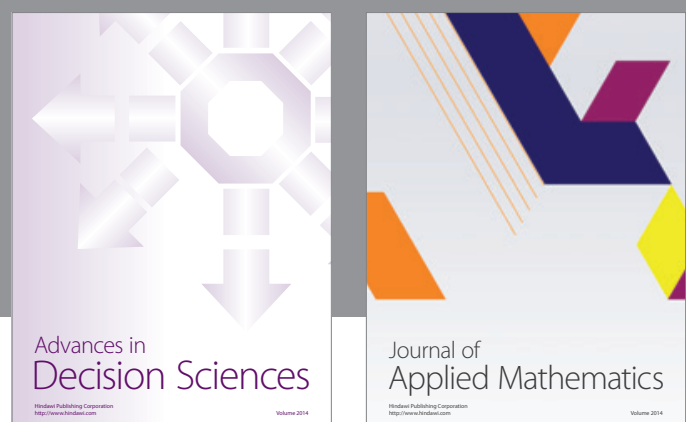

Journal of

Applied Mathematics
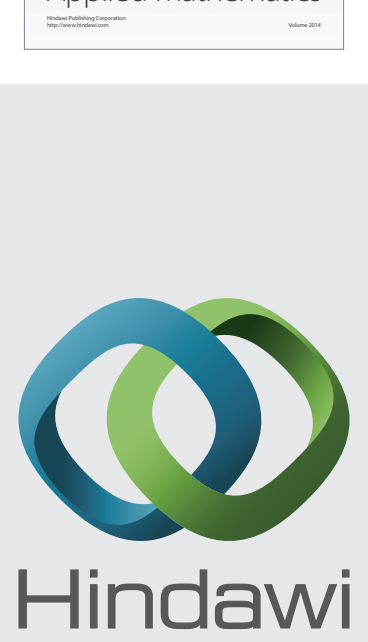

Submit your manuscripts at http://www.hindawi.com
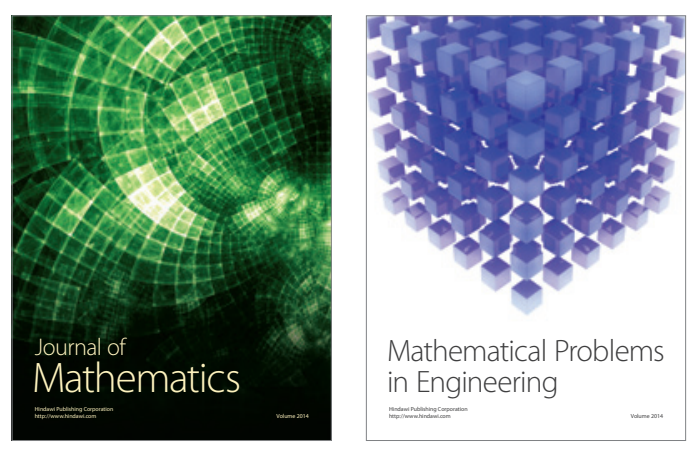

Mathematical Problems in Engineering
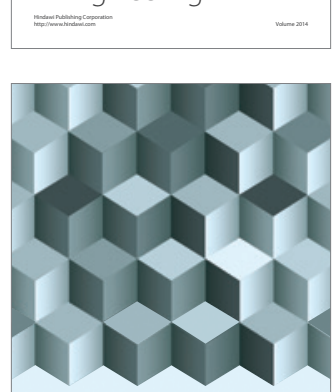

Journal of

Function Spaces
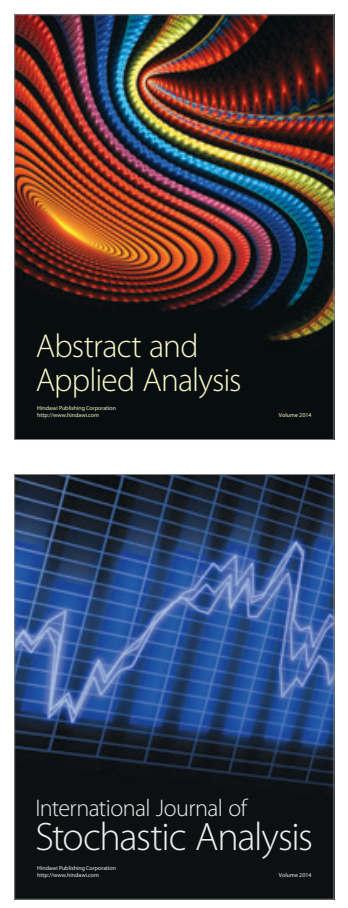

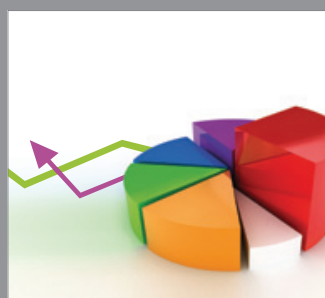

ournal of

Probability and Statistics

Promensencen
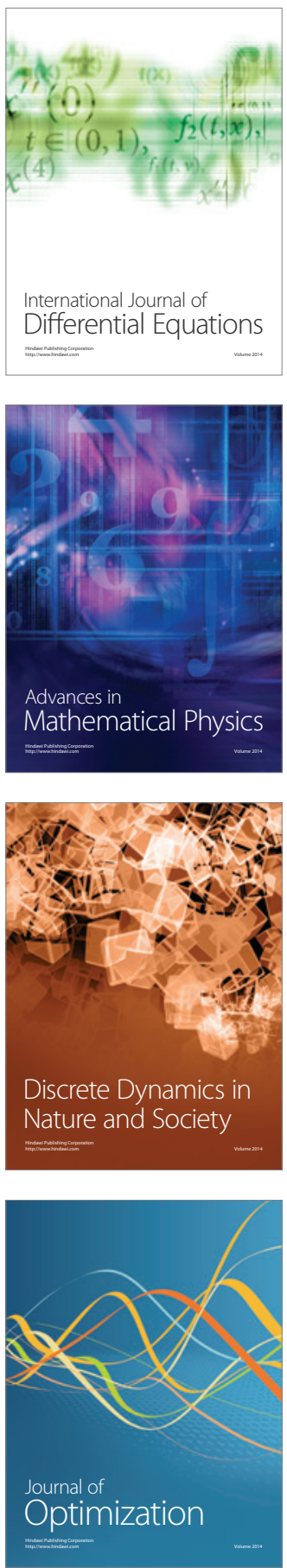